submitted to Journal of Mathematical Physics

\title{
Differential Galois obstructions for integrability of homogeneous Newton equations
}

\author{
Maria Przybylska
}

\begin{abstract}
Institut Fourier, UMR 5582 du CNRS, Université de Grenoble I, 100 rue des Maths, BP 74, 38402 Saint-Martin d'Hères Cedex, France, and

Toruń Centre for Astronomy, N. Copernicus University, Gagarina 11, PL-87-100 Toruń, Poland, (e-mail: Maria.Przybylska@astri.uni.torun.pl)
\end{abstract}

\begin{abstract}
In this paper we formulate necessary conditions for the integrability in the Jacobi sense of Newton equations $\ddot{\boldsymbol{q}}=-\boldsymbol{F}(\boldsymbol{q})$, where $\boldsymbol{q} \in \mathbb{C}^{n}$ and all components of $\boldsymbol{F}$ are polynomial and homogeneous of the same degree $l$. These conditions are derived from an analysis of the differential Galois group of the variational equations along special particular solutions of the Newton equations. We show that, taking all admissible particular solutions, we restrict considerably the set of Newton's equations satisfying the necessary conditions for the integrability. Moreover, we apply the obtained conditions for a detailed analysis of the Newton equations with two degrees of freedom (i.e., $n=2$ ). We demonstrate the strength of the obtained results analyzing general cases with $\operatorname{deg} F_{i}=l<4$. For $l=3$ we found an integrable case when the Newton equations have two polynomial first integrals and both of them are of degree four in the momenta $p_{1}=\dot{q}_{1}$, and $p_{2}=\dot{q}_{2}$. Moreover, for an arbitrary $k$, we found a family of Newton equations depending on one parameter $\lambda$. For an arbitrary value of $\lambda$ one quadratic in the momenta first integral exist. We distinguish infinitely many values of $\lambda$ for which the system is integrable or superintegrable with additional polynomial first integrals which seemingly can be of an arbitrarily high degree with respect to the momenta.
\end{abstract}

\section{Introduction}

In this paper we study the integrability of the following class of Newton's equations

$$
\ddot{\boldsymbol{q}}=-\boldsymbol{F}(\boldsymbol{q}), \quad \boldsymbol{q}=\left(q_{1}, \ldots, q_{n}\right)^{T} \in \mathbb{C}^{n} .
$$

In spite of the fact that such equations already appeared at the beginning of the mechanics in the Newton Principia [28], there is not too many rigorous results concerning their integrability. The only exception is the case of potential forces, i.e., the case when

$$
\boldsymbol{F}(\boldsymbol{q})=\frac{\partial V}{\partial \boldsymbol{q}}(\boldsymbol{q}),
$$


for a certain function $V$. In fact, for potential forces equations (1.1) are equivalent to Hamilton's equations

$$
\dot{\boldsymbol{q}}=\boldsymbol{p}, \quad \dot{\boldsymbol{p}}=-\frac{\partial V}{\partial \boldsymbol{q}}(\boldsymbol{q})
$$

with natural Hamiltonian

$$
H=\frac{1}{2} \boldsymbol{p}^{T} \boldsymbol{p}+V(\boldsymbol{q})
$$

First of all, for Hamiltonian systems we have a precise notion of the integrability i.e. the integrability in the Liouville sense. Moreover, for such systems we have powerfull methods and tools for integrability studies. This is why in this paper we investigate the integrability problem for Newton's equations with non-potential forces. However, for such equations 'integrability' is not well defined, so we should first specify what, in the considered context, the integrability means.

The oldest notion of the integrability is related to quadratures. We say that the considered system of differential equations is integrable by quadratures if all its solutions can be obtained in a closed form by a finite number of algebraic operations (including inversion of functions) and quadratures, i.e. integrals of 'known' functions, see e.g. [2]. In fact, already in his Principia Newton reduced to quadratures the simplest problems of dynamics (see theorems XXXIX, XLI, LIII and so on, see also comments in [8]). A similar definition of integrability was applied by Poincaré [29].

The integrability by quadratures was a prototype for other definitions of integrability. As a matter of fact, many of these new definitions can be considered as necessary conditions for the integrability by quadratures. Generally, if for a given dynamical system

$$
\dot{\boldsymbol{x}}=\boldsymbol{v}(\boldsymbol{x}), \quad \boldsymbol{x}=\left(x_{1}, \ldots x_{n}\right)^{T} \in \mathbb{C}^{n},
$$

there exists a sufficient number of tensor invariants, i.e. tensor fields that are constant along phase curves, then the system is solvable by quadratures. The most known two examples are the following. If system (1.4) possesses $n-1$ functionally independent first integrals, then it is integrable by quadratures. The Lie theorem says that if system (1.4) has $n$ symmeties, i.e., if there exist vector fields $\boldsymbol{u}_{1}(\boldsymbol{x})=\boldsymbol{v}(\boldsymbol{x}), \boldsymbol{u}_{2}(\boldsymbol{x}), \ldots, \boldsymbol{u}_{n}(\boldsymbol{x})$ which are linearly independent and $\left[\boldsymbol{u}_{i}, \boldsymbol{u}_{j}\right]=\mathbf{0}$, for $i, j=1, \ldots, n$, then system (1.4) is integrable by quadratures.

In [13] C. G. J. Jacobi developed, introduced by L. Euler, a method of the integrable factor. The Jacobi Last Multiplier of equation (1.4) is an invariant $n$-form

$$
\mu=h(\boldsymbol{x}) \mathrm{d} x_{1} \wedge \cdots \wedge \mathrm{d} x_{n} .
$$

Invariance of $\mu$ means that

$$
\operatorname{div}(h \boldsymbol{v}):=\sum_{i=1}^{n} \frac{\partial}{\partial x_{i}}\left(h v_{i}\right)=0 .
$$

Theorem 1.1 (Jacobi). Assume that system (1.4) possesses $n-2$ functionally independent first integrals and a Last Jacobi Multiplier. Then it is integrable by quadratures. 
We can also recall the well known fact that the integrability of a Hamiltonian system in the Liouville sense implies its integrability by quadratures, see e.g. [1]. For more details about the integrability by quadratures see 2 ; 7 ].

For the purpose of this paper we introduce the following definition.

Definition 1. We say that system (1.4) is integrable in the Jacobi sense if it admits an invariant $n$-form and possesses $n-2$ functionally independent first integrals.

This definition of the integrability is very often applied for nonholonomic systems, see e.g. [2; 7; 17; 5; 14].

Let us notice that Newton's equations (1.1) can be written in the following form

$$
\dot{\boldsymbol{q}}=\boldsymbol{p}, \quad \dot{\boldsymbol{p}}=-\boldsymbol{F}(\boldsymbol{q}),
$$

so they admit

$$
\mu=\mathrm{d} q_{1} \wedge \cdots \wedge \mathrm{d} q_{n} \wedge \mathrm{d} p_{1} \wedge \cdots \wedge \mathrm{d} p_{n}
$$

as an invariant $2 n$-form. Thus, in a case when equations (1.5) are not Hamiltonian, we say that they are integrable if they are integrable in the Jacobi sense.

The integrability does not depend on the chosen coordinates, however, the form of equations (1.5) and the homogeneity of $\boldsymbol{F}$ do. A linear change of variables given by $\boldsymbol{q}=A \boldsymbol{Q}, \boldsymbol{p}=A \boldsymbol{P}$, where $A \in \mathrm{GL}(n, \mathbb{C})$, transforms equations (1.5) into

$$
\dot{\boldsymbol{Q}}=\boldsymbol{P}, \quad \dot{\boldsymbol{P}}=-\boldsymbol{F}_{A}(\boldsymbol{Q}), \quad \text { where } \quad \boldsymbol{F}_{A}(\boldsymbol{Q}):=A^{-1} \boldsymbol{F}(A \boldsymbol{Q})
$$

Hence it is reasonable to divide all forces into disjoint equivalence classes. We say that forces $\boldsymbol{F}$ and $\boldsymbol{G}$ are equivalent iff there exists $A \in \mathrm{GL}(n, \mathbb{C})$ such that $\boldsymbol{G}=\boldsymbol{F}_{A}$. Later, specifying a force we just give a representative of equivalent forces in the above sense.

Our main purpose is to consider Newton's equations which are not Hamiltonian. Having this in mind, we introduce the following definition.

Definition 2. We say that a force $\boldsymbol{F}$ is potential iff there exists $A \in \operatorname{GL}(n, \mathbb{C})$ and $V \in \mathbb{C}[\boldsymbol{q}]$ such that $\boldsymbol{F}_{A}=\nabla V$.

For Hamiltonian systems there are many approaches to the integrability problem: the Hamilton-Jacobi theory, perturbation techniques, normal forms, splitting of separatrices and, recently, the Morales-Ramis theory based on differential Galois theory. Many integrable Hamiltonian systems were found and exhaustively analyzed by means of these methods. For non-Hamiltonian systems we have not so many various approaches.

The main aim of this paper is to formulate necessary conditions of integrability in the Jacobi sense for Newton systems (1.5). In this aim, we use an approach which is based on an investigation of variational equations for a particular complexified solution. This approach was developed by by S.L. Ziglin [35; 36] for Hamiltonian systems. However, the basic idea of his method can be used for a study of the integrability of an arbitrary dynamical system. The fact that the considered system possesses a meromorphic first integral implies that there exists a rational function which is an invariant of the monodromy group of the variational equations. This gives a restriction on the monodromy group. 
For the first time this method was applied for proving the non-integrability of a nonHamiltonian system by S.L. Ziglin in $[37 ; 38 ; 40 ; 39]$.

The Ziglin theory appeared to be a very strong tool for proving the non-integrability of Hamiltonian systems. Thanks to works of A. Baider, R. .C. Churchill, J. J. Morales, J.-P. Ramis, D. L. Rod, C. Simó and M. F. Singer the Ziglin theory was considerably developed, see $[4 ; 24 ; 25 ; 26]$ and references therein. The main idea of this development is following. As in the Ziglin theory, we investigate the variational equations along a nonequilibrium solution. But, instead of the monodromy group of the variational equations, we investigate their differential Galois group. This change gives the substantial profit because it is much easier to investigate the differential Galois group of the given equations than their monodromy group, and the differential Galois group contains the monodromy group as a subgroup. The main theorem due to Morales and Ramis states that if a Hamiltonian systems is integrable in the Liouville sense, then the identity component of the differential Galois group of variational equations along a non-equilibrium solution is Abelian. Some parts of the described differential Galois approach to the integrability can be adopted to non-Hamiltonian systems. The key implication is the following. If a system possesses a meromorphic first integral, then the differential Galois group of variational equations along a non-equilibrium solution has a rational invariant. In [19] the reader finds the first application of the differential Galois approach for proving non-integrability of a non-Hamiltonian system. This type of the integrability analysis was also applied for a nonholonomic system called the Suslov system in [21]. In this paper we apply the differential Galois approach for integrability studies of Newton's equations.

To formulate the main results of this paper we have to fix our assumptions. Hence, if it is not otherwise stated, we assume that force $\boldsymbol{F}=\left(F_{1}, \ldots, F_{n}\right)$ is polynomial and homogeneous, i.e., $F_{i} \in \mathbb{C}[\boldsymbol{q}]$, and $\operatorname{deg} F_{i}=l>1$ for $i=1, \ldots, n$. Moreover, we always write $\operatorname{deg} F_{i}=l=k-1$, and letter $k$ is used only in this context (if $\boldsymbol{F}$ is a potential force, then $k$ is the degree of the potential). Thus we have always $k>2$. By a direction in $\mathbb{C}^{n}$ we understand an equivalence class of parallel non-zero vectors. Of course a direction is determined just by one non-zero vector.

Definition 3. A direction $\boldsymbol{d} \in \mathbb{C}^{n}$ is a Darboux point of a force $\boldsymbol{F}(\boldsymbol{q})$, iff $\boldsymbol{d}$ is parallel to $\boldsymbol{F}(\boldsymbol{d})$, i.e., $\boldsymbol{d} \wedge \boldsymbol{F}(\boldsymbol{d})=\mathbf{0}$, and $\boldsymbol{F}(\boldsymbol{d}) \neq \mathbf{0}$.

To find all Darboux points of a given force it is enough to find all non-zero solutions of equations

$$
\boldsymbol{F}(\boldsymbol{d})=\boldsymbol{d},
$$

and take those which give different directions. To simplify exposition, we always use such normalization that a Darboux point $\boldsymbol{d}$ of $\boldsymbol{F}$ satisfies the above equation. The set of all Darboux points of a given force $\boldsymbol{F}$ is denoted by $\mathcal{D}_{\boldsymbol{F}}$. It can be an empty, infinite or finite set. If $\mathcal{D}_{\boldsymbol{F}}$ is finite, then for a generic $\boldsymbol{F}$ it has $D(n, k):=\left[(k-1)^{n}-1\right] /(k-2)$ elements.

A Darboux point $\boldsymbol{d}$ defines a particular solution of system (1.5) by

$$
\boldsymbol{q}(t)=\varphi(t) \boldsymbol{d}, \quad \boldsymbol{p}(t)=\dot{\varphi}(t) \boldsymbol{d},
$$

where $\varphi(t)$ satisfies the equation

$$
\ddot{\varphi}=-\varphi^{k-1} .
$$


This equation determines a family of hyperelliptic curves

$$
\dot{\varphi}^{2}=\frac{2}{k}\left(\varepsilon-\varphi^{k}\right),
$$

depending on a parameter $\varepsilon \in \mathbb{C}^{\star}$.

The variational equations along solution (1.8) have the form

$$
\dot{\boldsymbol{x}}=\boldsymbol{y}, \quad \dot{\boldsymbol{y}}=-\varphi(t)^{k-2} \boldsymbol{F}^{\prime}(\boldsymbol{d}) \boldsymbol{x},
$$

or simply

$$
\ddot{\boldsymbol{x}}=-\varphi(t)^{k-2} \boldsymbol{F}^{\prime}(\boldsymbol{d}) \boldsymbol{x},
$$

where $\boldsymbol{F}^{\prime}(\boldsymbol{d})$ is the Jacobi matrix of $\boldsymbol{F}$ calculated at a Darboux point $\boldsymbol{d}$. Let us assume that this matrix is diagonalizable. Then, in an appropriate basis equations (1.11) have the form

$$
\ddot{\eta}_{i}=-\lambda_{i} \varphi(t)^{k-2} \eta_{i}, \quad i=1, \ldots, n,
$$

where $\lambda_{1}, \ldots, \lambda_{n}$ are eigenvalues of $\boldsymbol{F}^{\prime}(\boldsymbol{d})$. By homogeneity of $\boldsymbol{F}$ one of the eigenvalues, let us say $\lambda_{n}$, is $(k-2)$. We denote the rest of eigenvalues by $\boldsymbol{\lambda}=\boldsymbol{\lambda}(\boldsymbol{d})=\left(\lambda_{1}, \ldots, \lambda_{n-1}\right) \in$ $\mathbb{C}^{n-1}$.

Our first theorem is the following.

Theorem 1.2. Assume that the Newton system (1.1) with polynomial homogeneous righthand sides of degree greater than one is integrable in the Jacobi sense. If $\boldsymbol{d}$ is a Darboux point of $\boldsymbol{F}$, such that $\boldsymbol{F}^{\prime}(\boldsymbol{d})$ is semi-simple, then the identity component of the differential Galois group of variational equations along the particular solution defined by $\boldsymbol{d}$ is Abelian.

Our next theorem gives a computable criterion for the integrability in the Jacobi sense.

Theorem 1.3. Assume that the Newton system (1.1) with polynomial homogeneous righthand sides of degree $l=k-1>1$ is integrable in the Jacobi sense. If $\boldsymbol{d}$ is a Darboux point of $\boldsymbol{F}$, such that $\boldsymbol{F}^{\prime}(\boldsymbol{d})$ is semi-simple, and $\lambda_{1}, \ldots, \lambda_{n}$ are eigenvalues of $\boldsymbol{F}^{\prime}(\boldsymbol{d})$, then $\left(k, \lambda_{i}\right)$ for $i=1, \ldots, n$ belong to the following list

case $k \quad \lambda$

$$
\begin{array}{llll}
\text { 1. } & k & p+\frac{k}{2} p(p-1) & \\
\text { 2. } & k & \frac{1}{2}\left(\frac{k-1}{k}+p(p+1) k\right) \\
\text { 3. } & 3 & -\frac{1}{24}+\frac{1}{6}(1+3 p)^{2}, & -\frac{1}{24}+\frac{3}{32}(1+4 p)^{2} \\
& & -\frac{1}{24}+\frac{3}{50}(1+5 p)^{2}, & -\frac{1}{24}+\frac{3}{50}(2+5 p)^{2} \\
\text { 4. } & 4 & -\frac{1}{8}+\frac{2}{9}(1+3 p)^{2} & \\
\text { 5. } & 5 & -\frac{9}{40}+\frac{5}{18}(1+3 p)^{2}, & -\frac{9}{40}+\frac{1}{10}(2+5 p)^{2}
\end{array}
$$


where $p$ is an integer. Moreover, among $\lambda_{1}, \ldots \lambda_{n}$ at most two belong to the first item of the above table.

The above theorem follows from Theorem 1.2 and the fact that each of the second order variational equations in (1.12) can be transformed into a Gauss hypergeometric equation for which the differential Galois group is known.

Our next result is of a different nature. It is easy to understand that an application of Theorem 1.3 to a general multi-parameter family of $\boldsymbol{F}$ gives rather an unsatisfactory result: we distinguish infinitely many families of $\boldsymbol{F}$ that satisfy conditions of Theorem 1.3. One can try to find more than one Darboux point and apply Theorem 1.3 to all of them. But in practice, without any guiding idea, an analysis of the obtained conditions is at least difficult.

To describe our approach, let us define for a Darboux point $\boldsymbol{d}$ of $\boldsymbol{F}$ quantities $\Lambda_{i}=$ $\lambda_{i}-1$, for $i=1, \ldots, n-1$, where $\lambda_{i}$ are eigenvalues of $\boldsymbol{F}^{\prime}(\boldsymbol{d})$. We denote them by $\boldsymbol{\Lambda}=\boldsymbol{\Lambda}(\boldsymbol{d})=\left(\Lambda_{1}, \ldots, \Lambda_{n-1}\right) \in \mathbb{C}^{n-1}$. Let $\tau_{i}$, for $i=0, \ldots, n-1$, denote elementary symmetric polynomials in $(n-1)$ variables. The following theorem shows that for a given $\boldsymbol{F}$, quantities $\boldsymbol{\Lambda}(\boldsymbol{d})$ calculated at different Darboux points are not arbitrary. It gives $n$ relations among $\boldsymbol{\Lambda}(\boldsymbol{d})$ calculated over all Darboux points $\boldsymbol{d} \in \mathcal{D}_{\boldsymbol{F}}$.

Theorem 1.4. Assume that $\boldsymbol{F}$ has exactly $D(n, k)$ Darboux points $\boldsymbol{d} \in \mathcal{D}_{\boldsymbol{F}}$. Then $\boldsymbol{\Lambda}(\boldsymbol{d})$ satisfy the following relations:

$$
\sum_{\boldsymbol{d} \in \mathcal{D}_{\boldsymbol{F}}} \frac{\tau_{1}(\boldsymbol{\Lambda}(\boldsymbol{d}))^{r}}{\tau_{n-1}(\boldsymbol{\Lambda}(\boldsymbol{d}))}=(-1)^{n}(n+k-2)^{r},
$$

or, alternatively

$$
\sum_{\boldsymbol{d} \in \mathcal{D}_{\boldsymbol{F}}} \frac{\tau_{r}(\boldsymbol{\Lambda}(\boldsymbol{d}))}{\tau_{n-1}(\boldsymbol{\Lambda}(\boldsymbol{d}))}=(-1)^{n-r-1} \sum_{i=0}^{r}\left(\begin{array}{c}
n-i-1 \\
r-i
\end{array}\right)(k-1)^{i},
$$

for $0 \leq r \leq n-1$.

The most important consequence of this theorem is the following. We show that for a fixed $k$, the set of solutions of (1.14) or (1.15), such that for each $\boldsymbol{d} \in \mathcal{D}_{\boldsymbol{F}}$ all components of $\boldsymbol{\lambda}(\boldsymbol{d})$ satisfy conditions of Theorem 1.3 is finite. Moreover, there is an algorithmic way to find all of those solutions. In other words, the above theorem gives a possibility to investigate the integrability of a general polynomial Newton system with a fixed degree of homogeneity.

It is worth mentioning here that the main idea which allows to derive the result contained in Theorem 1.4 is to consider the following auxiliary system

$$
\frac{\mathrm{d}}{\mathrm{d} t} \boldsymbol{q}=\boldsymbol{F}(\boldsymbol{q})
$$

associated with Newton's equations (1.5). It is a homogeneous first order system, so we can perform the Kovalevskaya analysis for it, see e.g. [17]. Then it appears that the 
introduced quantities $\Lambda_{i}$ are the Kovalevskaya exponents calculated at Darboux points of system (1.16). For more details, see Section 5 .

Without doubt the integrability is not a generic phenomenon. Thus one would like to weaken the assumptions of Theorem 1.4 and consider forces $\boldsymbol{F}$ with a smaller number of Darboux points. We do not know how to treat such cases for an arbitrary $n$, but for $n=2$ we present an approach which allows to formulate a theorem much stronger than Theorem 1.4, for details see Section 6.

Applications of our general theorems for Newton's equations with two degrees of freedom enabled us to distinguish for an arbitrary $k$ a one parameter family for which the necessary integrability conditions formulated in Theorem 1.3 are seemingly sufficient. An analysis given in Section 7.4 supports the following conjecture.

Conjecture 1.1. Assume that $(k, \lambda)$ belongs to an item of table (1.13). Then Newton equations

$$
\dot{q}_{1}=p_{1}, \quad \dot{p}_{1}=-\lambda q_{1} q_{2}^{k-2} \quad \dot{q}_{2}=p_{2}, \quad \dot{p}_{2}=q_{2}^{k-1}
$$

are integrable in the Jacobi sense with polynomial first integrals $I_{1}$ and $I_{2}$, where

$$
I_{1}=\frac{1}{2} p_{2}^{2}+\frac{1}{k} q_{2}^{k}
$$

Moreover, for an arbitrary $M>0$ we find $\lambda$ such that the degree of $I_{2}$ with respect to momenta is greater than $M$ and there is no an additional polynomial first integral independent with $I_{1}$ and degree with respect momenta smaller or equal $M$. Additionally, if $(k, \lambda)$ belongs to an item different from 1 in table (1.13), then there exist two additional polynomial first integrals $I_{2}$ and $I_{3}$ which are functionally independent together with $I_{1}$.

The plan of this paper is the following. In Section 2 definitions and some general results concerning the relations between first integrals of dynamical systems and invariants of their differential Galois group are presented. In Section 3 particular solutions and variational equations are calculated. Section 4 is crucial because contains necessary conditions of the Jacobi integrability. In Section 5 consequences of the existence of more particular solutions related to Darboux points for the integrability analysis are considered. Section 6 is a compendium of all general results for systems of two Newton's equations. In the next section we apply these results to the general classes of two Newton's homogeneous equations with the right-hand sides of degree two and three and for such systems the integrability analysis is almost complete. Some partial general results for two Newton equations with higher degrees of homogeneity are also presented. Appendix A contains two approaches to the problem how to recognized that the analyzed Newton's system is Hamiltonian. In Appendix B the basic facts about higher order variational equations and their applications are shown. The necessary integrability conditions expressed by means of higher order variational equations appeared to be a very effective tool for the analysis of Hamilton equations. In this part of the paper we formulate a conjecture that they can be also applied for the Newton equations and examples show the validity of this hypothesis. 


\section{Basic facts from general theory}

Let us consider a system of differential equations

$$
\frac{\mathrm{d}}{\mathrm{d} t} \boldsymbol{x}=\boldsymbol{v}(\boldsymbol{x}), \quad \boldsymbol{x} \in U \subset \mathbb{C}^{n}, \quad t \in \mathbb{C},
$$

where $\boldsymbol{v}$ is a holomorphic vector field in the considered domain $U$. For a non-equilibrium particular solution $\varphi(t)$ we consider also the variational equations along $\varphi(t)$, i.e.,

$$
\frac{\mathrm{d}}{\mathrm{d} t} \boldsymbol{\xi}=A(t) \boldsymbol{\xi}, \quad A(t)=\frac{\partial \boldsymbol{v}}{\partial \boldsymbol{x}}(\boldsymbol{\varphi}(t))
$$

The considered particular solution $\varphi(t)$ defines a Riemann surface $\Gamma$ immersed in $\mathbb{C}^{n}$ with $t$ as a local parameter. The right hand sides of variational equations (2.2) depend in fact on a point $\Gamma$.

If $F: \mathbb{C}^{n} \supset W \rightarrow \mathbb{C}$ is a holomorphic function defined in a certain connected neighborhood $W$ of the solution $\varphi(t)$, then, by definition, the leading term $f$ of $F$ is the lowest order non-vanishing term of expansion

$$
F(\boldsymbol{\varphi}(t)+\boldsymbol{\xi})=F_{m}(\boldsymbol{\xi})+O\left(\|\boldsymbol{\xi}\|^{m+1}\right), \quad F_{m} \neq 0,
$$

i.e., $f(\boldsymbol{\xi}):=F_{m}(\boldsymbol{\xi})$. Note that $f(\boldsymbol{\xi})$ is a homogeneous polynomial of variables $\boldsymbol{\xi}=$ $\left(\xi_{1}, \ldots, \xi_{n}\right)$ of degree $m$; its coefficients are polynomials in $\varphi(t)$. If $F$ is a meromorphic function, then $F=P / Q$ for certain holomorphic functions $P$ and $Q$. In this case, the leading term $f$ of $F$ is defined as $f=p / q$, where $p$ and $q$ are leading terms of $P$ and $Q$, respectively. In this case $f(\boldsymbol{\xi})$ is a homogeneous rational function of $\boldsymbol{\xi}$. To express this fact more precisely we denote by $\mathbb{K}=\mathcal{M}(\Gamma)$ the field of meromorphic functions on $\Gamma$. Then $f$ is a rational function of $\xi_{1}, \ldots, \xi_{n}$ with coefficients in $\mathbb{K}$, i.e., $f \in \mathbb{K}(\boldsymbol{\xi}):=\mathbb{K}\left(\xi_{1}, \ldots, \xi_{n}\right)$.

It is not difficult to prove that if $F$ is a meromorphic first integral of equation (2.1), then its leading term $f$ is a first integral of variational equations (2.2). Moreover, if $F_{1}, \ldots, F_{k}$ are functionally independent meromorphic first integrals of (2.1), then, by the Ziglin Lemma, we can assume that their leading terms $f_{1}, \ldots, f_{k}$ are functionally independent first integrals of (2.2). For proofs and details see $[35 ; 3 ; 4 ; 24]$.

Let $\mathcal{G} \subset \mathrm{GL}(n, \mathbb{C})$ denote the differential Galois group of (2.2). If $f(g(\boldsymbol{\xi}))=f(\boldsymbol{\xi})$ for every $g \in \mathcal{G}$, then we say that $f$ is an invariant of $\mathcal{G}$. The following lemma says that first integrals of variational equations are invariants of their differential Galois group. It was proved in [24], see also Lemma III.1.13 on p.63 in [3].

Lemma 2.1. If equation (2.1) has $k$ functionally independent first integrals which are meromorphic in a connected neighborhood of a non-equilibrium solution $\varphi(t)$, then the differential Galois group $\mathcal{G}$ of the variational equations along $\varphi(t)$ has $k$ functionally independent rational invariants.

For a fixed $t \in \mathbb{C}$ (or, for a fixed point $\varphi(t)$ on the Riemann surface $\Gamma$ ), a first integral $f$ of the variational equations is an element of $\mathbb{C}(\boldsymbol{\xi})$. The set of all rational invariants of $\mathcal{G}$ is a field denoted by $\mathbb{C}(\boldsymbol{\xi})^{\mathcal{G}}$. 
The differential Galois group $\mathcal{G}$ of a system of linear equations is a linear algebraic group, see e.g. [15; 33]. So, in particular, it is also a Lie group. This fact allows to reformulate the necessary conditions for the integrability in the language of Lie algebras. However, passing from a Lie group to its Lie algebra, we have to pay something. Namely, we take into account only the identity component $\mathcal{G}^{0}$ of $\mathcal{G}$. In particular, if $\mathcal{G}$ is finite, then the Lie algebra of $\mathcal{G}$ is trivial, i.e., it consists of one zero vector.

Let $\mathfrak{g} \subset \mathrm{GL}(n, \mathbb{C})$ denote the Lie algebra of $\mathcal{G}$. An element $Y \in \mathfrak{g}$ can be considered as a linear vector field: $\boldsymbol{x} \mapsto Y(\boldsymbol{x}):=Y \boldsymbol{x}$, for $\boldsymbol{x} \in \mathbb{C}^{n}$. We say that $f \in \mathbb{C}(\boldsymbol{x})$ is an integral of $\mathfrak{g}$, iff $Y(f)(\boldsymbol{x})=\mathrm{d} f(\boldsymbol{x}) \cdot Y(\boldsymbol{x})=0$, for all $Y \in \mathfrak{g}$. All rational first integrals of the Lie algebra $\mathfrak{g} \subset \mathrm{GL}(n, \mathbb{C})$ form a field denoted by $\mathbb{C}(\boldsymbol{x})^{\mathfrak{g}}$.

Proposition 2.1. If $f_{1}, \ldots, f_{k} \in \mathbb{C}(\boldsymbol{x})$ are algebraically independent invariants of an algebraic group $\mathcal{G} \subset \mathrm{GL}(n, \mathbb{C})$, then they are algebraically independent first integrals of the Lie algebra $\mathfrak{g}$ of $\mathcal{G}$.

Proof. Let $X \in \mathfrak{g}$. Then $g(t)=\exp [t X]$ defines for $t \in \mathbb{R},|t|$ small enough, a one parameter subgroup of $\mathcal{G}$. Thus if $f \in \mathbb{C}(\boldsymbol{x})^{\mathcal{G}}$, then

$$
f(\exp [t X] \boldsymbol{x})=f(\boldsymbol{x}),
$$

for any $X \in \mathfrak{g}$. Differentiating the above equality with respect to $t$ at $t=0$, we obtain $X(f)=0$, i.e., $f \in \mathbb{C}(\boldsymbol{x})^{\mathfrak{g}}$.

It can happen that two systems, one integrable and the other non-integrable, have the same variational equations along a chosen particular solution. In such situation, to show non-integrability of the second system we have to apply either another method or another particular solution or investigate higher order variational equations. The last approach appears very effective. In this paper we use it analyzing several examples and it is described in Appendix B.

\section{Differential Galois group of variational equations}

We showed in Introduction that the variational equations (1.12) for a particular solution (1.8) corresponding to a Darboux point $\boldsymbol{d}$ are a direct product of second order equations of the form

$$
\ddot{\eta}=-\lambda \varphi(t)^{k-2} \eta, \quad \lambda \in \mathbb{C},
$$

where $\varphi(t)$ satisfies (1.9). Let $\mathcal{G}(k, \lambda) \subset \operatorname{Sp}(2, \mathbb{C})$ denote the differential Galois group of the above equation and $\mathcal{G}(k, \lambda)^{\circ}$ the identity component of $\mathcal{G}(k, \lambda)$. Then the differential Galois group $\mathcal{G}$ of variational equations (1.12) is a direct product

$$
\mathcal{G}=\mathcal{G}\left(k, \lambda_{1}\right) \times \cdots \times \mathcal{G}\left(k, \lambda_{n}\right) \subset \operatorname{Sp}(2 n, \mathbb{C}) .
$$

Hence, it is clear that in order to know the properties of $\mathcal{G}$, we should know as much as possible about $\mathcal{G}(k, \lambda)$. Fortunately, the properties of the last group can be described in 
details. Following Yoshida [34] it is convenient to define a new independent variable $z$ in (3.1) by

$$
z:=\frac{1}{\varepsilon} \varphi(t)^{k},
$$

where $\varphi(t)$ satisfies (1.9). Then equation (3.1) transforms into a linear second order equation with rational coefficients

$$
z(1-z) \eta^{\prime \prime}+\left(\frac{k-1}{k}-\frac{3 k-2}{2 k} z\right) \eta^{\prime}+\frac{\lambda_{i}}{2 k} \eta=0 .
$$

This equation is a special case of the Gauss hypergeometric equation

$$
z(1-z) \eta^{\prime \prime}+[c-(a+b+1) z] \eta^{\prime}-a b \eta=0,
$$

with

$$
a+b=\frac{k-2}{2 k}, \quad a b=-\frac{\lambda}{2 k}, \quad c=1-\frac{1}{k} .
$$

Thus, the differences of exponents at singularities $z=0,1$ and $\infty$ for equation (3.4) are

$$
\rho=1-c=\frac{1}{k}, \quad \sigma=c-a-b=\frac{1}{2}, \quad \tau=a-b=\frac{1}{2 k} \sqrt{(k-2)^{2}+8 k \lambda},
$$

respectively. Let $G(k, \lambda) \subset \mathrm{GL}(2, \mathbb{C})$ denote a differential Galois group of (3.3). The differential Galois group of the hypergeometric equation is well known, see e.g. [16; 12]. However, to make this fact usefull, we should know a relation between $\mathcal{G}(k, \lambda)$ and $G(k, \lambda)$. It can be shown, see Proposition 4.7 in [4], that the identity components of these groups are the same.

The well known Kimura theorem [16] specifies all cases when all solutions of the hypergeometric equation (3.4) are Liouvillian. In these cases, by the Lie-Kolchin theorem, the identity component of the differential Galois group is solvable. A direct application of the Kimura theorem to equation (3.3) gives the following.

Lemma 3.1. The identity component $G(k, \lambda)^{\circ}$ of the differential Galois group of equation (3.3) with $k>2$ is solvable if and only if $(k, \lambda)$ belong to the list given in Theorem 1.3.

The above lemma is not sufficient for our purpose. We show the following.

Lemma 3.2. Assume that $(k, \lambda)$ belongs to the list given in Theorem 1.3 and $k>2$. Then, for the differential Galois group $G(k, \lambda)$ of equation (3.3), the following statements hold.

1. $G(k, \lambda)^{\circ}$ is Abelian.

2. If $(k, \lambda)$ belongs to the item 1 of the list, then

$$
G(k, \lambda)^{\circ}=\mathcal{T}_{1}:=\left\{\left[\begin{array}{ll}
1 & c \\
0 & 1
\end{array}\right] \mid c \in \mathbb{C}\right\}
$$


3. If $(k, \lambda)$ belongs to items $2-5$ of the list, then $G(k, \lambda)^{\circ}=\{E\}$.

Here $E$ is the identity matrix.

Proof. At first we transform equation (3.3) into the normal form. To this end we put

$$
w=\eta \exp \left[\frac{1}{2} \int p \mathrm{~d} z\right], \quad p:=\frac{c-(a+b+1) z}{z(1-z)},
$$

where $a, b$ and $c$ are given by (3.5). Then we obtain

$$
w^{\prime \prime}=\frac{\rho^{2}-1+z\left(1-\rho^{2}-\tau^{2}+\sigma^{2}\right)+z^{2}\left(\tau^{2}-1\right)}{4 z^{2}(z-1)^{2}} w .
$$

For this equation exponents at 0,1 and at infinity are

$$
\left\{\frac{1}{2}(1-\rho), \frac{1}{2}(1+\rho)\right\}, \quad\left\{\frac{1}{2}(1-\sigma), \frac{1}{2}(1+\sigma)\right\}, \quad\left\{-\frac{1}{2}(1-\tau),-\frac{1}{2}(1+\tau)\right\},
$$

respectively. Monodromy and differential Galois groups of (3.8) are now subgroups of $\mathrm{SL}(2, \mathbb{C})$. It is important to remark here that the identity components of the differential Galois group of (3.3) and (3.8) are the same. Notice also that the differences of exponents at singular points were unchanged. We denote by $\widehat{G}(k, \lambda)$ the differential Galois group of equation (3.8).

By assumption $G(k, \lambda)^{\circ}$ is solvable, and thus $\widehat{G}(k, \lambda)^{\circ}$ is also solvable. Suppose that $\widehat{G}(k, \lambda)^{\circ}$ is solvable but not Abelian. Then, by Theorem 4.12 on p. 31 in [15] and Proposition 4.2 in [32], there is only one possibility: $\widehat{G}(k, \lambda)=\widehat{G}(k, \lambda)^{\circ}=\mathcal{T}$, where $\mathcal{T}$ is the triangular subgroup of $\mathrm{SL}(2, \mathbb{C})$. So, such case can appear only if the considered equation is reducible. Using the well known criterion for the reducibility of Riemann $P$ equation, see e.g. [16], we easily obtain that equation (3.8) is reducible iff $\lambda=p+k p(p-1) / 2$ where $p \in \mathbb{Z}$.

We show that if equation (3.8) is reducible, then $\widehat{G}(k, \lambda)$ is a proper subgroup of $\mathcal{T}$, and in this way we prove the first statement of our lemma.

First, let us notice that in the considered reducible case the respective exponents at singular points 0,1 and infinity are following

$$
\left\{\frac{1}{2}-\frac{1}{2 k}, \frac{1}{2}+\frac{1}{2 k}\right\}, \quad\left\{\frac{1}{4}, \frac{3}{4}\right\}, \quad\left\{-\frac{2+k(l+2)}{4 k}, \frac{2+k(l-2)}{4 k}\right\},
$$

where $l$ is an odd integer. The difference of exponents at the singular point $z=1$ is $1 / 2$. Thus, by Lemma 4.3.6 on p. 90 in [12] equation (3.8) has a solution of the form:

$$
w=z^{r}(1-z)^{s} h(z)
$$

where $h(z)$ is a polynomial, and $r$ is an exponent at $z=0$, and $s$ in an exponent at $z=1$, As $r$ and $s$ are rational, there exists $j \in \mathbb{N}$ such that $w^{j} \in \mathbb{C}(z)$. Now, by Proposition 4.2 in [32], $\widehat{\mathcal{G}}(k, \lambda)$ is either a proper subgroup of the diagonal group, or a proper subgroup of the triangular group. This finishes proof of the first statement of the lemma. 
To prove the second statement of we have to show that $\widehat{\mathcal{G}}(k, \lambda)$ cannot be a subgroup of the diagonal group. Assume that it is diagonal. Then, in particular, the monodromy group of equation (3.8) is diagonal. This last group is generated by two elements $M_{0}$, $M_{1} \in \mathrm{SL}(2, \mathbb{C})$ - the monodromy matrices corresponding to canonical loops encircling singular points 0 and 1 , respectively. If the monodromy group of equation (3.8) is diagonal, then by Lemma 4.3 .5 on p. 90 in [12], at least one of the matrices $M_{0}, M_{1}$ or $M_{0} M_{1}$ is a scalar matrix, i.e., $\pm E$. Taking into account, that the eigenvalues of $M_{i}$ are $\exp 2 \pi \mathrm{i} r_{i, 1}$ and $\exp 2 \pi \mathrm{i} r_{i, 1}$, where $r_{i, 1}$ and $r_{i, 2}$ are exponents at $z=i$, we easily conclude that for $k>2$ it is impossible. This finishes the proof of the second statement.

To show the last statement it is enough to notice that if $(k, \lambda)$ belongs to item 2 of the list (1.13), then, using Theorem 2.9 (b) on p. 525 in [6] one can check that $\widehat{G}(k, \lambda)$ is finite. Moreover, from the proof of the Kimura theorem given in [16] it follows that if $(k, \lambda)$ belongs to items $3-5$ of the list 1.13 , then $G(k, \lambda)$, and thus $\widehat{G}(k, \lambda)$, is a finite primitive group. As the identity component of a finite group is just the identity element, this finishes the proof.

Passing from group $G(k, \lambda)$ to its Lie algebra from the lemma proved above we have as an immediate consequence the following corollary.

Corollary 3.1. Let $\mathfrak{g}$ be the Lie algebra of the differential Galois group $G(k, \lambda)$ of equation (3.3). Assume that $(k, \lambda)$ belongs to the list given in Lemma 3.1 and $k>2$. If $(k, \lambda)$ belongs to the item 1 , then $\operatorname{dim}_{\mathbb{C}} \mathfrak{g}=1$, and otherwise $\operatorname{dim}_{\mathbb{C}} \mathfrak{g}=0$.

\section{Necessary conditions for the Jacobi integrability}

The main purpose of this section is to give proofs of Theorems 1.2 and 1.3 . For this purpose we develop appropriate tools investigating certain Lie algebras.

\subsection{Certain Poisson algebra}

An element $Y$ of Lie algebra $\operatorname{sp}(2 n, \mathbb{C})$, considered as a linear vector field, is a Hamiltonian vector field given by a global Hamiltonian function $H: \mathbb{C}^{2 n} \rightarrow \mathbb{C}$, which is a homogeneous polynomial of $2 n$ variables $\left(x_{1}, \ldots, x_{n}, y_{1}, \ldots, y_{n}\right)$ of degree 2 , i.e. $H \in \mathbb{C}_{2}[\boldsymbol{x}, \boldsymbol{y}]:=$ $\mathbb{C}_{2}\left[x_{1}, \ldots, x_{n}, y_{1}, \ldots, y_{n}\right]$. In this way, we identify Lie algebra $\operatorname{sp}(2 n, \mathbb{C})$ with a $\mathbb{C}$-linear vector space $\mathbb{C}_{2}[\boldsymbol{x}, \boldsymbol{y}]$ with the canonical Poisson bracket as the Lie bracket. Thus, for a Lie algebra $\mathfrak{g} \subset \operatorname{sp}(2 n, \mathbb{C}) \simeq \mathbb{C}_{2}[\boldsymbol{x}, \boldsymbol{y}]$, a rational function $f \in \mathbb{C}[\boldsymbol{x}, \boldsymbol{y}]$ is a first integral of $\mathfrak{g}$, iff $\{H, f\}=0$, for all $H \in \mathfrak{g}$.

Now, we consider a more detailed case when $\mathfrak{g}$ is a Lie subalgebra of $\operatorname{sp}(2, \mathbb{C})$. It is easy to show that Lie algebra $\operatorname{sp}(2, \mathbb{C})$ does not admit any non-constant first integral.

Proposition 4.1. A rational function $f \in \mathbb{C}(x, y)$ is a first integral of $\operatorname{sp}(2, \mathbb{C})$, iff $f \in \mathbb{C}$. Proof. Let $f \in \mathbb{C}(x, y)$ be a first integral of $\operatorname{sp}(2, \mathbb{C}) \simeq \mathbb{C}_{2}[x, y]$. Thus, $\{f, H\}=0$, for each $H \in \mathbb{C}_{2}[x, y]$. Let us take $H=x^{2}$. Then,

$$
\{f, H\}=-2 x \frac{\partial f}{\partial y}=0
$$


and this shows that $f$ does not depend on $y$, i.e., $f \in \mathbb{C}(x)$. Taking $H=y^{2}$, we show that $f$ does not depend on $x$. Hence $f \in \mathbb{C}$.

The above proposition shows that only proper subalgebras of $\operatorname{sp}(2, \mathbb{C})$ can have nonconstant first integrals.

Proposition 4.2. If $\mathfrak{g}$ is a Lie subalgebra of $\operatorname{sp}(2, \mathbb{C})$ and $\operatorname{dim}_{\mathbb{C}} \mathfrak{g}>0$, then the number of algebraically independent rational first integrals of $\mathfrak{g}$ is not greater than one.

Proof. As $\operatorname{dim}_{\mathbb{C}} \mathfrak{g}>0$, there exists a non-zero $H \in \operatorname{sp}(2, \mathbb{C}) \simeq \mathbb{C}_{2}[x, y]$. The number of rational algebraically independent first integrals of a non-zero linear Hamiltonian vector field $X_{H}$ in $\mathbb{C}^{2}$ is at most one.

Proposition 4.3. If $\mathfrak{g}$ is a Lie subalgebra of $\operatorname{sp}(2, \mathbb{C})$ and $\operatorname{dim}_{\mathbb{C}} \mathfrak{g}=2$, then $\mathbb{C}(x, y)^{\mathfrak{g}}=\mathbb{C}$.

Proof. All two dimensional Lie algebras are solvable, so $\mathfrak{g}$ is solvable. Thus a connected Lie group $G \subset \operatorname{sp}(2, \mathbb{C})$ with Lie algebra $\mathfrak{g}$ is solvable. By the Lie-Kolchin theorem $G$ is conjugate to the triangular group

$$
\mathcal{T}:=\left\{\left[\begin{array}{cc}
a & b \\
0 & a^{-1}
\end{array}\right] \mid a \in \mathbb{C}^{\star}, \quad b \in \mathbb{C}\right\} .
$$

The Lie algebra $\mathfrak{t}$ of $\mathcal{T}$ is isomorphic to $\mathfrak{g}$, and is generated by two elements

$$
h_{1}=\left[\begin{array}{cc}
1 & 0 \\
0 & -1
\end{array}\right], \quad h_{2}=\left[\begin{array}{ll}
0 & 1 \\
0 & 0
\end{array}\right] \text {. }
$$

Let $H_{1}$ and $H_{2}$ be Hamiltonian functions from $\mathbb{C}_{2}[x, y]$ such that linear vector fields $X_{H_{1}}$ and $X_{H_{2}}$ are represented by matrices $h_{1}$ and $h_{2}$, respectively. It is easy to check that

$$
H_{1}=x y, \quad H_{2}=\frac{1}{2} y^{2} .
$$

We show that $\mathbb{C}(x, y)^{\mathfrak{t}}=\mathbb{C}$. Assume that there exists $f \in \mathbb{C}(x, y)^{\mathfrak{t}} \backslash \mathbb{C}$. Hence $\left\{f, H_{i}\right\}=0$ for $i=1,2$. But

$$
\left\{f, H_{2}\right\}=y \frac{\partial f}{\partial x}=0
$$

so, $f \in \mathbb{C}(y)$. However, for $f \in \mathbb{C}(y)$, we have

$$
\left\{f, H_{1}\right\}=-y \frac{\partial f}{\partial y}=0
$$

and this implies that $f \in \mathbb{C}$. A contradiction with the assumption that $f$ is not a constant shows that $\mathbb{C}(x, y)^{\mathfrak{t}}=\mathbb{C}$. Moreover, as the Lie algebras $\mathfrak{t}$ and $\mathfrak{g}$ are isomorphic, we have also $\mathbb{C}(x, y)^{\mathfrak{g}}=\mathbb{C}$.

Let $\mathbb{K} \supset \mathbb{C}$ be a field. Then, for the Lie algebra $\operatorname{sp}(2, \mathbb{C}) \simeq \mathbb{C}_{2}[x, y]$, we can also consider first integrals which belong to $\mathbb{K}(x, y)$. Here we assume that

$$
\frac{\partial a}{\partial x}=0 \quad \text { and } \quad \frac{\partial a}{\partial y}=0 \text { for all } a \in \mathbb{K} .
$$

For our further considerations we need the following lemma. 
Lemma 4.1. Let $\mathfrak{g}$ be a one dimensional Lie subalgebra of $\operatorname{sp}(2, \mathbb{C}) \simeq \mathbb{C}_{2}[x, y]$. If $f \in$ $\mathbb{K}(x, y)$ is a rational first integral of $\mathfrak{g}$, then there exists a nonzero element $h \in \mathbb{C}[x, y]$ such that $f \in \mathbb{K}(h)$.

Proof. Let us assume that $\mathfrak{g}$ is nilpotent, i.e., $\mathfrak{g}$ is generated by $H=y^{2}$. Then we have

$$
0=\{H, f\}=-2 y \frac{\partial f}{\partial x} .
$$

Thus, $f$ does not depend on $x$, so for this case we choose $h=y$.

The only other possibility is that $\mathfrak{g}$ is diagonal, i.e., it is generated by $H=x y$. First, let us assume that $f$ is a polynomial in $(x, y)$. We can write $f$ uniquely as a sum of homogeneous components. It is clear that if $f$ is the first integral of $H$, then each homogeneous component of $f$ is also the first integral of $H$. Thus, let us assume that $f$ is homogeneous of degree $s$ and let us represent it in the form

$$
f=\sum_{i=0}^{s} f_{i} x^{i} y^{s-i}, \quad f_{i} \in \mathbb{K} \quad \text { for } \quad i=1, \ldots, s .
$$

Then we obtain

$$
0=\{H, f\}=\sum_{i=0}^{s} f_{i}(s-i) x^{i} y^{s-i}-\sum_{i=0}^{s} i f_{i} x^{i} y^{s-i}=\sum_{i=0}^{s} f_{i}(s-2 i) x^{i} y^{s-i} .
$$

Hence, $f_{i}=0$ for $2 i \neq s$, and if for even $s=2 r, f_{r} \neq 0$, then $f=f_{r}(x y)^{r}$. This implies that every homogeneous, and thus arbitrary polynomial first integral $f \in \mathbb{K}[x, y]$ of $H$, is an element of $\mathbb{K}[h]$, where $h=x y$.

Now, assume that $f$ is a rational first integral of $H$. Then we can write $f=P / Q$ where $P$ and $Q$ are relatively prime polynomials in $\mathbb{K}[x, y]$. Hence we have

$$
0=\{H, P / Q\}=\frac{1}{Q^{2}}(Q\{H, P\}-P\{H, Q\}),
$$

so $Q\{H, P\}=P\{H, Q\}$. As $P$ and $Q$ are relatively prime, this implies that

$$
\{H, P\}=\gamma P \quad \text { and } \quad\{H, Q\}=\gamma Q .
$$

for a certain $\gamma \in \mathbb{K}[x, y]$. Comparing degrees of both sides in the above equalities, we deduce that $\gamma \in \mathbb{K}$. If $\gamma=0$, then $P$ and $Q$ are polynomial first integrals of $H$, so in this case we have that $f \in \mathbb{K}(h)$.

We show that the case $\gamma \neq 0$ is impossible. Let us assume that $\gamma \neq 0$. It is easy to see that if $P \in \mathbb{K}[x, y]$ satisfies equation

$$
\{H, P\}=\gamma P,
$$

then its every homogeneous component also satisfies this equation. Thus let us assume that $P$ is homogeneous of degree $s$. If we write

$$
P=\sum_{i=0}^{s} P_{i} x^{i} y^{s-i}, \quad P_{i} \in \mathbb{K} \quad \text { for } \quad i=1, \ldots, s,
$$


then equation (4.6) leads to the following equality

$$
\sum_{i=0}^{s} P_{i}(s-2 i-\gamma) x^{i} y^{s-i}=0 .
$$

This implies that if coefficient $P_{i} \neq 0$, then $\gamma=s-2 i$ and $P=P_{i} x^{i} y^{s-i}$. Thus, every homogeneous solution of (4.6) is a monomial of the form $P_{i} x^{i} y^{i+\gamma}$, where $\gamma$ is a non-zero integer and $i$ is a non-negative integer such that $i+\gamma \geq 0$. Thus a non-homogeneous solution of (4.6) is a finite sum

$$
P=\sum_{i+\gamma>0} p_{i} x^{i} y^{i+\gamma}
$$

But $Q$ satisfies the same equation (4.6), so we also have

$$
Q=\sum_{j+\gamma>0} q_{j} x^{j} y^{j+\gamma}
$$

If $\gamma>0$, then $P$ and $Q$ are not relatively prime, because they have a common factor $y^{\gamma}$. On the other hand, if $\gamma<0$, then they are not relatively prime, either, because they have a common factor $x$. We have a contradiction and this finishes the proof.

Let us denote by $\mathfrak{s}$ the Lie algebra which is the direct sum of $n$ copies of $\operatorname{sp}(2, \mathbb{C})$

$$
\mathfrak{s}:=\underbrace{\operatorname{sp}(2, \mathbb{C}) \oplus \cdots \oplus \operatorname{sp}(2, \mathbb{C})}_{n \text {-times }}
$$

and by $\pi_{i}: \mathfrak{s} \rightarrow \operatorname{sp}(2, \mathbb{C})$, the projection onto the $i$-th component of $\mathfrak{s}$, for $i=1, \ldots, n$.

Lemma 4.2. Let $\mathfrak{g}$ be a Lie subalgebra of $\mathfrak{s}$, and $\mathfrak{g}_{i}=\pi_{i}(\mathfrak{g})$ for $i=1, \ldots, n$. Assume that $\mathfrak{g}$ has $2 n-2$ rational algebraically independent first integrals. If for some $1 \leq j \leq n$, $\operatorname{dim}_{\mathbb{C}} \mathfrak{g}_{j}>1$, then $\mathfrak{g}_{i}=\{0\}$, for $i \neq j$.

Proof. We consider elements of Lie algebra $\mathfrak{s}$ as degree two homogeneous polynomials of $2 n$ variables $(\boldsymbol{x}, \boldsymbol{y})$. Because $\mathfrak{s}$ is a direct sum of the form (4.9), we have

$$
\mathfrak{s} \simeq \bigoplus_{i=1}^{n} \mathbb{C}_{2}\left[x_{i}, y_{i}\right]
$$

and an element $H \in \mathfrak{s}$ admits a unique representation

$$
H=\sum_{i=1}^{n} H_{i}, \quad H_{i} \in \mathbb{C}_{2}\left[x_{i}, y_{i}\right], \quad \text { for } \quad i=1, \ldots, n .
$$

Without loss of generality, we prove our lemma for $j=n$. Let us assume that $\operatorname{dim}_{\mathbb{C}} \mathfrak{g}_{n}>1$, and let $f \in \mathbb{C}(\boldsymbol{x}, \boldsymbol{y}) \backslash \mathbb{C}$ be a first integral of $\mathfrak{g}$. Then from the proofs of Propositions 4.2 
and 4.3 it follows that $f$ does not depend on $\left(x_{n}, y_{n}\right)$. Thus, if $\mathfrak{g}$ has $2 n-2$ algebraically independent rational first integrals $f_{i}$, then

$$
f_{i} \in \mathbb{C}\left(x_{1}, \ldots, x_{n-1}, y_{1}, \ldots, y_{n-1}\right) \backslash \mathbb{C}, \quad i=1, \ldots, 2 n-2 .
$$

Assume that there exists $0<i<n$ such that $\mathfrak{g}_{i} \neq 0$. Then there exists a non-zero $H \in \mathbb{C}_{2}\left[x_{i}, y_{i}\right]$ such that

$$
\left\{f_{j}, H\right\}=\frac{\partial f_{j}}{\partial x_{i}} \frac{\partial H}{\partial y_{i}}-\frac{\partial f_{j}}{\partial y_{i}} \frac{\partial H}{\partial x_{i}}=0, \quad \text { for } \quad j=1, \ldots 2 n-2 .
$$

Hence, the linear Hamiltonian vector field $X_{H}$ considered as a vector field in $\mathbb{C}^{2 n-2}$ with coordinates $\left(x_{1}, \ldots, x_{n-1}, y_{1}, \ldots, y_{n-1}\right)$ has $2 n-2$ first integrals (4.10) and so $X_{H}=0$. Thus we have a contradiction because for a non-zero $H \in \mathbb{C}_{2}\left[x_{i}, y_{i}\right], X_{H} \neq 0$.

Lemma 4.3. Let $\mathfrak{g}$ be an Abelian Lie subalgebra of $\mathfrak{s}$, and $\mathfrak{g}_{i}=\pi_{i}(\mathfrak{g})$ for $i=1, \ldots, n$. Assume that $\mathfrak{g}$ has $2 n-2$ rational algebraically independent first integrals. Then $\operatorname{dim}_{\mathbb{C}} \mathfrak{g} \leq$ 2 .

Proof. As $\mathfrak{g}$ is Abelian, $\mathfrak{g}_{i}$ is Abelian, and $\operatorname{dim}_{\mathbb{C}} \mathfrak{g}_{i} \leq 1$ for $i=1, \ldots, n$. Let $\operatorname{dim}_{\mathbb{C}} \mathfrak{g}=s$. Then among $\mathfrak{g}_{i}$, exactly $s$ have dimension 1 , and the remaining are zero-dimensional. We can assume that $\operatorname{dim}_{\mathbb{C}} \mathfrak{g}_{i}=1$ for $i=1, \ldots, s$, and $\operatorname{dim}_{\mathbb{C}} \mathfrak{g}_{i}=0$ for $i=s+1, \ldots, n$.

Let $f \in \mathbb{C}(\boldsymbol{x})^{\mathfrak{g}}$, then, in particular $f$ is a first integral of $\mathfrak{g}_{1}$. We can consider $f$ as an element of $\mathbb{K}\left(x_{1}, y_{1}\right)$, where $\mathbb{K}=\mathbb{C}\left(x_{2}, \ldots, x_{n}, y_{2}, \ldots, y_{n}\right)$. Then, by Lemma 4.1. there exists $h_{1} \in \mathbb{C}\left(x_{1}, y_{1}\right)$ such that $f \in \mathbb{K}\left(h_{1}\right)=\mathbb{C}\left(h_{1}, x_{2}, \ldots, x_{n}, y_{2}, \ldots, y_{n}\right)$. Repeating successively the above reasoning for $\mathfrak{g}_{i}$, for $i=2, \ldots, s$, we obtain that $f$ is an element of $R:=\mathbb{C}\left(h_{1}, \ldots, h_{s}, x_{s+1}, \ldots, x_{n}, y_{s+1}, \ldots, y_{n}\right)$, where $h_{i} \in \mathbb{C}\left(x_{i}, y_{i}\right) \backslash \mathbb{C}$, for $i=1, \ldots, s$. Thus a first integral of $\mathfrak{g}$ depends on $r=s+2(n-s)=2 n-s$ variables. This implies that every set of $p>r$ elements from $R$ is algebraically dependent. Hence, if $f_{1}, \ldots, f_{p}$ are algebraically independent elements of $R$, (i.e. algebraically independent first integrals of $\mathfrak{g}$ ), then $p \leq r$. So, from assumption of our lemma $p=2 n-2 \leq 2 n-s$, and thus $s \leq 2$.

\subsection{Proofs of Theorems 1.2 and 1.3}

Under assumption of both theorems, the differential Galois group of the variational equations is a direct product (3.2). Thus we also have

$$
\mathcal{G}^{\circ}=\mathcal{G}\left(k, \lambda_{1}\right)^{\circ} \times \cdots \times \mathcal{G}\left(k, \lambda_{n}\right)^{\circ} \subset \operatorname{Sp}(2 n, \mathbb{C}) .
$$

Moreover, the Lie algebra $\mathfrak{g}$ is a direct sum

$$
\mathfrak{g}=\bigoplus_{i=1}^{n} \mathfrak{g}_{i}, \quad \mathfrak{g}_{i} \in \operatorname{sp}(2, \mathbb{C})
$$

We have already remarked that the identity components of the differential Galois groups of equations (3.1), (3.3) and (3.8) are the same. Hence, conclusions of Lemma 3.1 and Lemma 3.2 are valid for $\mathcal{G}(k, \lambda)^{\circ}$. 
We prove Theorem 1.2 by contradiction. Let us assume that $\mathcal{G}^{\circ}$ is not Abelian. Then there exists $1 \leq i \leq n$ such that $\mathcal{G}\left(k, \lambda_{i}\right)^{\circ}$ is not Abelian. We have two possibilities. Either $\mathcal{G}\left(\lambda_{i}\right)^{\circ}$ is solvable and in this case it conjugates to the whole triangular group of $\operatorname{Sp}(2, \mathbb{C})$, or it is not solvable and then it is $\operatorname{Sp}(2, \mathbb{C})$, see Proposition 2.2 in 24]. In both cases dimension of Lie algebra $\mathfrak{g}_{i}$ of $\mathcal{G}\left(k, \lambda_{i}\right)^{\circ}$ is at least 2. Now, by Lemma 4.2, for $r \neq i$, Lie algebras $\mathfrak{g}_{r}$ of groups $\mathcal{G}\left(k, \lambda_{r}\right)$ are zero dimensional.

We know that among $\lambda_{j}$ one, let us say $\lambda_{s}, s \in\{1, \ldots, n\}$, equals $k-1$, thus it belongs to the item 1 of the table (1.13). For $\lambda=\lambda_{s}$ the identity component $G(k, \lambda)^{\circ}$ of differential Galois group of hypergeometric equation (3.3) is Abelian but its Lie algebra has dimension one, see Lemma 3.2. But $G(k, \lambda)^{\circ}=\mathcal{G}(k, \lambda)^{\circ}$, so Lie algebra $\mathfrak{g}_{s}$ has dimension one. It follows that $s \neq i$. But it is impossible because we have shown that for each $k \neq i$, $k \in\{1, \ldots, n\}$, Lie algebras $\mathfrak{g}_{k}$ are zero dimensional. A contradiction finishes the proof of Theorem 1.2

Now, we prove Theorem 1.3. From Theorem (1.2) it follows that $\mathcal{G}^{0}$ is Abelian and thus $\mathcal{G}\left(k, \lambda_{i}\right)^{\circ}$ are Abelian for $i=1, \ldots, n$. As $\mathcal{G}\left(k, \lambda_{i}\right)^{\circ}=G\left(k, \lambda_{i}\right)^{\circ}$, we can apply Lemma 3.1 and we obtain immediately the first statement of Theorem 1.3, The last statement of this theorem follows from Lemma 4.3 and Corollary 3.1.

\section{Global analysis}

The aim of this section is to present a proof of Theorem 1.4. The central role in our considerations plays a theorem proved in [11], see also [10]. This theorem is related to the Kovalevskaya and Painlevé analysis. To formulate it we recall basic facts.

Let

$$
\boldsymbol{e}(\boldsymbol{x}):=\sum_{i=1}^{n} x_{i} \frac{\partial}{\partial x_{i}}, \quad \boldsymbol{x}=\left(x_{1}, \ldots, x_{n}\right),
$$

be the Euler vector field. A polynomial vector field $\boldsymbol{v}$ given by

$$
\boldsymbol{v}(\boldsymbol{x}):=\sum_{i=1}^{n} v_{i}(\boldsymbol{x}) \frac{\partial}{\partial x_{i}}, \quad v_{i} \in \mathbb{C}[\boldsymbol{x}], \quad i=1, \ldots, n,
$$

is called homogeneous of degree $p$, iff $L_{\boldsymbol{e}}(\boldsymbol{v})=p \boldsymbol{v}$, where $L_{\boldsymbol{e}}$ denotes the Lie derivative along the Euler field. Notice that a polynomial vector field $\boldsymbol{v}$ is homogeneous of degree $p$ iff $v_{i}$ are homogeneous and

$$
\operatorname{deg} v_{i}=p+1 \quad \text { for } \quad i=1, \ldots, n \text {. }
$$

With vector field (5.2) we associate the following polynomial differential equation

$$
\frac{\mathrm{d}}{\mathrm{d} t} \boldsymbol{x}=\boldsymbol{v}(\boldsymbol{x}), \quad \boldsymbol{x} \in \mathbb{C}^{n} .
$$

In this context, abusing notation, we consider $\boldsymbol{v}$ as an element of $\mathbb{C}[\boldsymbol{x}]^{n}$. We say that system (5.3) is homogeneous of degree $p$ iff $\boldsymbol{v}$ as a vector field is homogeneous of degree $p$. 
Definition 4. We say that a direction $\boldsymbol{d} \in \mathbb{C}^{n}$ is a Darboux point of a homogeneous vector field $\boldsymbol{v}$, or a Darboux point of system (5.3), iff $\boldsymbol{e}(\boldsymbol{d}) \wedge \boldsymbol{v}(\boldsymbol{d})=\mathbf{0}$ and $\boldsymbol{v}(\boldsymbol{d}) \neq \mathbf{0}$.

Thus vector field $\boldsymbol{v}$ and the Euler field are parallel at a Darboux point. This implies that there exists a unique $\rho \in \mathbb{C}^{\star}$ such that vector field $\rho \boldsymbol{v}-\boldsymbol{e}$ has an equilibrium point at $\boldsymbol{d}$. To find all Darboux points of a given vector field $\boldsymbol{v}$, it is enough to find all non-zero solutions of non-linear equations

$$
\boldsymbol{v}(\boldsymbol{d})=\boldsymbol{d},
$$

and distinguish among them those giving different directions. We always assume that a Darboux point $\boldsymbol{d}$ is normalized in such a way that it satisfies (5.4). Notice also that a Darboux point $\boldsymbol{d}=\left(d_{1}, \ldots, d_{n}\right)$ can be considered as a point of the projective space $\mathbb{C P}^{n-1}$ with homogeneous coordinates $\left[d_{1}: \cdots: d_{n}\right]$.

The set of all Darboux points of a vector field $\boldsymbol{v}$ we denote by $\mathcal{D}_{\boldsymbol{v}}$. It can be empty, finite or infinite. One can show, see Proposition 4 in [11], that if all Darboux points of a homogeneous vector field $\boldsymbol{v}$ of degree $p$ are isolated, then their number is not greater than $d(n, p):=\left((p+1)^{n}-1\right) / p$, and a generic $\boldsymbol{v}$ has exactly this number of Darboux points.

Let $\boldsymbol{d}$ be a Darboux point of a homogeneous vector field (5.2). The Kovalevskaya matrix $K(\boldsymbol{d})$ at point $\boldsymbol{d}$ is defined as

$$
K(\boldsymbol{d}):=\boldsymbol{v}^{\prime}(\boldsymbol{d})-E
$$

and its eigenvalues are called the Kovalevskaya exponents.

REMARK 5.1 In the above definition we assumed that Darboux point $\boldsymbol{d}$ satisfies (5.4). Without this assumption the Kovalevskaya matrix at Darboux point $\boldsymbol{d}$ is defined as the matrix of linearization of vector field $\rho \boldsymbol{v}-\boldsymbol{e}$ at equilibrium point $\boldsymbol{d}$, i.e.,

$$
K(\boldsymbol{d}):=\rho \boldsymbol{v}^{\prime}(\boldsymbol{d})-E .
$$

We have the following fact.

Proposition 5.1. Let $\boldsymbol{d} \in \mathbb{C}^{n}$ be a Darboux point of homogeneous vector field $\boldsymbol{v}$ of degree $p$. Then $\boldsymbol{d}$ is an eigenvector of the Kovalevskaya matrix $K(\boldsymbol{d})$, and the corresponding eigenvalue is $p$.

An easy proof of the above proposition we leave to the reader.

Let $\Lambda_{i}=\Lambda_{i}(\boldsymbol{d})$ for $i=1, \ldots, n$ denote the Kovalevskaya exponents at a Darboux point $\boldsymbol{d} \in \mathcal{D}_{\boldsymbol{v}}$. By Proposition [5.1, one of them is $p$. We always assume that $\Lambda_{n}=p$, and denote by $\boldsymbol{\Lambda}=\boldsymbol{\Lambda}(\boldsymbol{d})=\left(\Lambda_{1}, \ldots, \Lambda_{n-1}\right) \in \mathbb{C}^{n-1}$. Let $\tau_{i}$ for $0 \leq i \leq n-1$, denote the elementary symmetric polynomial in $(n-1)$ variables of degree $i$. In paper [11] it was shown that the values of $\boldsymbol{\Lambda}(\boldsymbol{d})$ taken at different Darboux points are not arbitrary, i.e., there exist universal relations among all $\boldsymbol{\Lambda}(\boldsymbol{d}), \boldsymbol{d} \in \mathcal{D}_{\boldsymbol{v}}$.

Theorem 5.1. Assume that a homogeneous polynomial vector field $\boldsymbol{v}$ of degree $p>0$ has $d(n, p)$ isolated Darboux points, and let $S$ be a symmetric homogeneous polynomial in $n-1$ variables of degree lower than $n$. Then, the number

$$
R:=\sum_{\boldsymbol{d} \in \mathcal{D}_{\boldsymbol{v}}} \frac{S(\boldsymbol{\Lambda}(\boldsymbol{d}))}{\tau_{n-1}(\boldsymbol{\Lambda}(\boldsymbol{d}))},
$$


depends only on a choice of $S$, the dimension $n$ and the degree $p$.

In other words, $R=R(S, n, p)$ does not depend on a specific choice of $\boldsymbol{v}$, provided that $\boldsymbol{v}$ has the maximal number of Darboux points $d(n, p)$. For applications of the above theorem we have to know values $R(S, n, p)$ for an arbitrary $n, p$, and for a chosen set of independent symmetric polynomials $S$ of degree not greater than $n-1$. The standard choice for $S$ are elementary symmetric polynomials $\tau_{i}$, or alternatively $\tau_{1}^{i}$, for $0 \leq i \leq n-1$. To calculate $R(S, n, p)$ it is enough to find a homogeneous system defined for an arbitrary $n>2$ and $p \geq 0$ possessing the maximal number of Darboux points, and such that one can determine the Kovalevskaya exponents easily for all its Darboux points. These requirements are satisfied be a $n$-dimensional generalization of the Jouanolou system [18]

$$
\dot{x}_{i}=x_{i+1}^{p+1}, \quad 1 \leq i \leq n, \quad x_{n+1} \equiv x_{i} .
$$

For this system, the Kovalevskaya exponents at all Darboux points are the same and are given by

$$
\Lambda_{i}=(p+1) \varepsilon^{n-i}-1, \quad 1 \leq i \leq n,
$$

where $\varepsilon$ is a primitive $n$-th root of the unity. In [31] we have shown that

$$
\tau_{r}(\boldsymbol{\Lambda})=(-1)^{r} \sum_{i=0}^{r}\left(\begin{array}{c}
n-i-1 \\
r-i
\end{array}\right)(p+1)^{i},
$$

for $0 \leq r \leq n-1$. Using this fact one can show that

$$
R\left(\tau_{1}^{r}, n, p\right)=(-1)^{n+r-1}(n+p)^{r},
$$

and

$$
R\left(\tau_{r}, n, p\right)=(-1)^{n-i-1} \sum_{i=0}^{r}\left(\begin{array}{c}
n-i-1 \\
r-i
\end{array}\right)(p+1)^{i},
$$

for $0 \leq r \leq n-1$.

Now we return to the Newton equations (1.5). We associate with them the following auxiliary system

$$
\frac{\mathrm{d}}{\mathrm{d} t} \boldsymbol{x}=\boldsymbol{F}(\boldsymbol{x}), \quad \boldsymbol{x} \in \mathbb{C}^{n} .
$$

As $\operatorname{deg} F_{i}=k-1$ for $i=1, \ldots n, \boldsymbol{F}$ considered as a vector field has degree $p=k-2$. A Darboux point of force $\boldsymbol{F}$ is a Darboux point of the auxiliary system. Thus, if $\boldsymbol{d}$ is a Darboux point of (5.12), then among the Kovalevskaya exponents calculated at $\boldsymbol{d}$, one is $k-2$. We denote the remaining ones by $\boldsymbol{\Lambda}(\boldsymbol{d})=\left(\Lambda_{1}(\boldsymbol{d}), \ldots, \Lambda_{n-1}(\boldsymbol{d})\right)$. Assuming that the auxiliary system has $d(n, k-2)=D(n, k)$ isolated Darboux points we can apply Theorem 5.1, Then, taking into account relations (5.10) and (5.11), we obtain the thesis of Theorem 1.4 and this finishes its proof.

One of the most important consequences of Theorem 1.4 is the following. Let $\mathcal{F}(n, k)$ denote a set of forces with exactly $L=D(n, k)$ Darboux points $\boldsymbol{d}_{j}, j=1, \ldots, L$. For each 
$\boldsymbol{F} \in \mathcal{F}(n, k)$ we have $\kappa=(n-1) L$ nontrivial Kovalevskaya exponents. We denote their collection by $\mathcal{K}(\boldsymbol{F}) \in \mathbb{C}^{\kappa}$, i.e.,

$$
\mathcal{K}(\boldsymbol{F}):=\left(\boldsymbol{\Lambda}\left(\boldsymbol{d}_{1}\right), \ldots, \boldsymbol{\Lambda}\left(\boldsymbol{d}_{L}\right)\right) .
$$

If for $\mathcal{K}(\boldsymbol{F})$ given above, all $\left(k, \lambda_{i, j}\right), \lambda_{i, j}=\Lambda_{i}\left(\boldsymbol{d}_{j}\right)+1$ for $i=1, \ldots, n-1$ and $j=1, \ldots, L$ belong to table (1.13) in Theorem 1.3, then we write $\mathcal{K}(\boldsymbol{F}) \in \mathcal{J}(n, k)$. In other words, if for $\boldsymbol{F} \in \mathcal{F}(n, k)$ the corresponding Newton's equations are integrable in the Jacobi sense, then $\mathcal{K}(\boldsymbol{F}) \in \mathcal{J}(n, k)$, and if $\mathcal{K}(\boldsymbol{F}) \in \mathcal{J}(n, k)$, then the Newton equations satisfy the necessary conditions for the integrability in the Jacobi sense of Theorem 1.3. We show that sets $\mathcal{J}(n, k)$ are finite.

Theorem 5.2. For $n, k \geq 2$ set $\mathcal{J}(n, k)$ is finite.

Proof. Relation (1.15) in Theorem 1.4 for $r=n-2$ and $p=k-2$ reads

$$
\sum_{j=1}^{L} \sum_{i=1}^{n-1} \frac{1}{\Lambda_{i}\left(\boldsymbol{d}_{j}\right)}=-\frac{(k-1)^{n}-n(k-2)-1}{(k-2)^{2}} .
$$

By definition of $\mathcal{J}(n, k)$, quantities $\lambda_{i, j}=\Lambda_{i}\left(\boldsymbol{d}_{j}\right)+1$ for $i=1, \ldots, n-1$ and $j=1, \ldots, L$ belong to sets listed in table (1.13) in Theorem 1.3. Because of this, we can apply directly Lemma B.1 from 22] and this finishes the proof. See also the proof of Theorem 4 in [31].

\section{Two degrees of freedom}

In this section we investigate in detail the case of the Newton equations with two degrees of freedom. The main aim is to show that in this case we can obtain more general results than those given by Theorem 1.4. Moreover, we investigate also the case of forces which admit infinitely many Darboux points, as well as cases when forces do not have Darboux points.

Before we start our analysis of the system with two degrees of freedom, we make two general remark. If a force admits Darboux points $\boldsymbol{d}_{1}, \ldots, \boldsymbol{d}_{l}, 1 \leq l \leq n$, then we can choose $l$ coordinate axes along them. In a such choosen frame in such all but $j$-th coordinates of $\boldsymbol{d}_{j}$ are zero.

Newton's system of the form (1.5) contains as a subclass natural Hamiltonian systems. Thus it is convenient to have a simple criterion to distinguish such systems. In Appendix A we present two methods that enable to check the equivalence of the analyzed Newton system to a Hamiltonian one.

In the rest of this section we assume that $\boldsymbol{F}=\left(F_{1}, F_{2}\right)$, and we put

$$
F_{j}=\sum_{i=0}^{k-1} F_{i}^{j} q_{1}^{k-1-i} q_{2}^{i}, \quad \text { for } \quad j=1,2 .
$$




\subsection{Darboux Points}

Darboux points of a force $\boldsymbol{F}$ are the equilibria of the vector field $\boldsymbol{G}(\boldsymbol{q})=\boldsymbol{F}(\boldsymbol{q})-\boldsymbol{q}$. Thus, it is convenient to introduce an auxiliary system

$$
\dot{q}_{1}=-q_{1}+F_{1}, \quad \dot{q}_{2}=-q_{2}+F_{2} .
$$

However, we must remember that different equilibria of the above system can correspond to the same Darboux point. To cope with this problem we introduce coordinates $x=q_{1}$ and $z=q_{2} / q_{1}$ and define

$$
f_{i}(z):=F_{i}(1, z) \quad \text { for } \quad i=1,2 .
$$

Then the auxiliary system (6.2) in these coordinates takes the form

$$
\dot{x}=-x+x^{k-1} h(z), \quad \dot{z}=x^{k-2} g(z),
$$

where

$$
h(z)=f_{1}(z), \quad g(z)=f_{2}(z)-z f_{1}(z) .
$$

Notice that $\operatorname{deg} g \leq k$. As it has been mentioned, a Darboux point is a point in a projective space, and here it is $\mathbb{C P}^{1}$ with $\left[q_{1}: q_{2}\right]$ as homogeneous coordinates. Hence, $z$ is a coordinate on the affine part of $\mathbb{C P}^{1}$. An equilibrium of (6.4) is a solution of

$$
x^{k-2} h(z)=1, \quad x^{k-2} g(z)=0, \quad x \neq 0,
$$

and thus the Darboux points are roots of polynomial $g(z)$ such that $h(z)$ does not vanish on them. This immediately implies that either the number of Darboux points is infinite when $g=0$, or it is finite and not greater than $k$. We say that a Darboux point is multiple iff its $z$ coordinate is a multiple root of $g(z)$.

REMARK 6.1 As it has already been mentioned, not all roots of $g(z)$ are Darboux points. If $g\left(z_{\star}\right)=h\left(z_{\star}\right)=0$, then $z_{\star}$ is not a Darboux point. Equations $g\left(z_{\star}\right)=h\left(z_{\star}\right)=0$ imply that $f_{1}\left(z_{\star}\right)=f_{2}\left(z_{\star}\right)=0$, i.e., $F_{1}$ and $F_{2}$ have a common factor $P=q_{2}-z_{\star} q_{1}$.

If $[0: 1] \in \mathbb{C P}^{1}$ is a Darboux point, then we say that it is located at the infinity. To investigate its neighborhood we use coordinates $y=q_{2}$ and $\zeta=q_{1} / q_{2}$. We define

$$
\tilde{f}_{i}(\zeta):=F_{i}(\zeta, 1), \quad \text { for } \quad i=1,2,
$$

and

$$
\tilde{g}(\zeta):=\tilde{f}_{1}(\zeta)-\zeta \tilde{f}_{2}(\zeta), \quad \tilde{h}(\zeta):=\tilde{f}_{2}(\zeta) .
$$

Then a Darboux point is a root of polynomial $\tilde{g}$, such that it is not a root of $\tilde{h}$. If $\tilde{g}(0)=\tilde{f}_{1}(0)=0$ and simultaneously $\tilde{h}(0)=\tilde{f}_{2}(0) \neq 0$, then the infinity is a Darboux point. Notice that

$$
\tilde{f}_{i}(\zeta)=\zeta^{k-1} f_{i}\left(\frac{1}{\zeta}\right) \quad \text { for } \quad i=1,2 .
$$


In many cases, it is convenient to assume that all Darboux points are located in the affine part of $\mathbb{C P}^{1}$, i.e., that there is no Darboux point at the infinity. If $\operatorname{deg} g=k$, then surely the infinity is not a Darboux point. Hence, let us analyze whether we can assume that for a given force the corresponding polynomial $g$ is of degree $k$. From the definition of polynomial $g$, see (6.5), it follows that $\operatorname{deg} g=k$ if and only if $\operatorname{deg} f_{1}=k-1$. We show the following.

Proposition 6.1. Let $\boldsymbol{F}=\left(F_{1}, F_{2}\right), F_{1}, F_{2} \in \mathbb{C}\left[q_{1}, q_{2}\right]$ be a homogeneous force and $\operatorname{deg} F_{i}=k-1$ for $i=1,2$. Assume that for each $A \in \mathrm{GL}(2, C)$ for the equivalent force $\boldsymbol{F}_{A}=\left(F_{1}^{A}, F_{2}^{A}\right)$ we have $\operatorname{deg}_{q_{2}} F_{1}^{A}<k-1$. Then

$$
G:=q_{1} F_{2}-q_{2} F_{1}=0 .
$$

Proof. One can directly check that if $\boldsymbol{F}$ satisfies condition (6.10), then every force equivalent to $\boldsymbol{F}$ also satisfies this condition.

First let us notice that condition $\operatorname{deg}_{q_{2}} F_{1}<k-1$ is equivalent to $\operatorname{deg} f_{1}<k-1$, and this is equivalent to $\tilde{f}_{1}(0)=0$. In fact, if

$$
f_{1}(z)=\sum_{i=0}^{l} f_{i}^{1} z^{i}, \quad f_{l}^{1} \neq 0 \quad \text { and } \quad l<k-1,
$$

then

$$
\tilde{f}_{1}(\zeta)=\zeta^{k-1} f_{1}\left(\frac{1}{\zeta}\right)=\sum_{i=0}^{l} f_{i}^{1} \zeta^{k-1-i}
$$

and hence $\tilde{f}_{1}(0)=0$.

Let

$$
A=\left[\begin{array}{cc}
\alpha & \beta \\
\gamma & \delta
\end{array}\right], \quad \Delta=\alpha \delta+\beta \gamma \neq 0
$$

Then

$$
F_{1}^{A}\left(q_{1}, q_{2}\right)=\frac{1}{\Delta}\left[\delta F_{1}\left(\alpha q_{1}+\beta q_{2}, \gamma q_{1}+\delta q_{2}\right)-\beta F_{2}\left(\alpha q_{1}+\beta q_{2}, \gamma q_{1}+\delta q_{2}\right)\right],
$$

and hence

where

$$
\tilde{f}_{1}^{A}(\zeta)=\frac{1}{\Delta}(\gamma \zeta+\delta)^{k-1}\left[\delta \tilde{f}_{1}(\tau(\zeta))-\beta \tilde{f}_{2}(\tau(\zeta))\right]
$$

$$
\tau(\zeta)=\frac{\alpha \zeta+\beta}{\gamma \zeta+\delta}
$$

Assume that $\delta \neq 0$ and denote $x=\beta / \delta$. Then we obtain

$$
\tilde{f}_{1}^{A}(0)=\frac{\delta^{k}}{\Delta}\left[\tilde{f}_{1}(x)-x \tilde{f}_{2}(x)\right]=\frac{\delta^{k}}{\Delta} \tilde{g}(x) .
$$

By assumption $\tilde{f}_{1}^{A}(0)=0$ for an arbitrary $A$, so

$$
\tilde{f}_{1}(x)-x \tilde{f}_{2}(x)=0
$$

for an arbitrary $x \in \mathbb{C}$. Putting $x=q_{1} / q_{2}$ in the above equality an using the definition of $\tilde{f}_{1}$ and $\tilde{f}_{2}$, see (6.7), we obtain condition (6.10). 
Remark 6.2 Condition (6.10) means that force $\boldsymbol{F}=\left(F_{1}, F_{2}\right)$ admits infinitely many Darboux points.

\subsection{Generic case}

Now we determine the Kovalevskaya exponents at a given Darboux point. The Jacobian matrix of the auxiliary system (6.4) has the form

$$
J=\left[\begin{array}{cc}
-1+(k-1) x^{k-2} h(z) & x^{k-1} h^{\prime}(z) \\
(k-2) x^{k-3} g(z) & x^{k-2} g^{\prime}(z)
\end{array}\right]
$$

For a Darboux point $(x, z)$ satisfying (6.6) this matrix becomes

$$
J=\left[\begin{array}{cc}
k-2 & x h^{\prime}(z) / h(z) \\
0 & g^{\prime}(z) / h(z)
\end{array}\right]
$$

Hence the non-trivial Kovalevskaya exponent at a Darboux point with coordinate $z$ is given by

$$
\Lambda(z)=\frac{g^{\prime}(z)}{h(z)}=\frac{f_{2}^{\prime}(z)-f_{1}(z)-z f_{1}^{\prime}(z)}{f_{1}(z)} .
$$

On the projective line $\mathbb{C P}^{1}$, where Darboux points are located, we define a meromorphic differential form $\omega$ which in coordinate $z$ is given by

$$
\omega=\frac{h(z)}{g(z)} \mathrm{d} z
$$

and in coordinate $\zeta=1 / z$ in the neighborhood of infinity by

$$
\omega_{\infty}=-\frac{h(1 / \zeta)}{g(1 / \zeta)} \frac{\mathrm{d} \zeta}{\zeta^{2}}
$$

Notice that if $z_{\star}$ is a Darboux point, then it is a pole of $\omega$, and the residue of $\omega$ at this point is

$$
\operatorname{res}\left(\omega, z_{\star}\right)=\frac{h\left(z_{\star}\right)}{g^{\prime}\left(z_{\star}\right)}=\frac{1}{\Lambda\left(z_{\star}\right)},
$$

provided that $g$ and $h$ are relatively prime and $z_{\star}$ is a simple root of $g$.

For a given force $\boldsymbol{F}=\left(F_{1}, F_{2}\right)$ with finitely many Darboux points, we can always assume that all of them are located in the affine part of $\mathbb{C P}^{1}$ i.e. for all Darboux points $q_{1} \neq 0$. This can be achieved by a proper choice of coordinates. Under this assumption we have $F_{1}\left(0, q_{2}\right) \neq 0$, and hence $F_{k-1}^{1} \neq 0$, see (6.1). We keep this assumption in our further considerations.

To formulate a generalization of Theorem 1.4 we have to distinguish a class of forces for which this is possible. Let $\mathcal{A}_{l}$ denote a set of forces $\boldsymbol{F}=\left(F_{1}, F_{2}\right)$ satisfying the following conditions

A.1 $\boldsymbol{F}$ admits $1 \leq l \leq k$ simple Darboux points; 
A.2 if $F_{1}(\boldsymbol{q})$ and $G(\boldsymbol{q}):=q_{2} F_{1}(\boldsymbol{q})-q_{1} F_{2}(\boldsymbol{q})$ have a common linear factor $P=\alpha q_{1}+\beta q_{2}$, $|\alpha|+|\beta| \neq 0$, then the multiplicity of $P$ in $G$ is not greater than the multiplicity of $P$ in $F_{1}$.

Theorem 6.1. Assume that $\boldsymbol{F} \in \mathcal{A}_{l}$, and let $\Lambda_{i}$ is the non-trivial Kovalevskaya exponent of $\boldsymbol{F}$ at $i$-th Darboux point for $i=1, \ldots, l$. Then

$$
\sum_{i=1}^{l} \frac{1}{\Lambda_{i}}=-1
$$

Proof. We can assume that $\operatorname{deg} g(z)=k$ and $\operatorname{deg} h(z)=k-1$. Moreover, from the definitions of $h$ and $g$, see (6.5), it follows that if $\alpha z^{k-1}$ is the highest order term of $h$, then $-\alpha z^{k}$ is the highest order term of $g$.

Although for $l=k$ the statement of theorem coincides with Theorem 1.4, it is instructive to give first an independent proof for this case. Thus, let $k=l$. Then $g$ and $h$ are relatively prime and all $k$ roots $z_{i}$ of $g$ are simple. We have

$$
\operatorname{res}\left(\omega, z_{i}\right)=\frac{h\left(z_{i}\right)}{g^{\prime}\left(z_{i}\right)}=\frac{1}{\Lambda_{i}} \quad \text { for } \quad i=1, \ldots, k .
$$

To calculate the residue of $\omega$ at infinity, i.e., $\operatorname{res}\left(\omega_{\infty}, 0\right)$, we notice that for polynomials $\tilde{h}(\zeta)=\zeta^{k-1} h(1 / \zeta)$ and $\tilde{g}(\zeta)=\zeta^{k} g(1 / \zeta)$ we have $\tilde{h}(0)=\alpha$ and $\tilde{g}(0)=-\alpha$. Thus

$$
-\frac{h(1 / \zeta)}{\zeta^{2} g(1 / \zeta)}=-\frac{\tilde{h}(\zeta)}{\zeta \tilde{g}(\zeta)}=\frac{1}{\zeta}+\cdots
$$

where dots denote higher order terms. This shows that $\operatorname{res}\left(\omega_{\infty}, 0\right)=1$ and the application of the global residue theorem, see e.g. [9], finishes the proof for $l=k$.

For $1 \leq l<k$ the assumption that $\boldsymbol{F} \in \mathcal{A}_{l}$ implies that $g(z)$ has $l$ simple roots $z_{1}, \ldots, z_{l}$, such that $h\left(z_{i}\right) \neq 0$ for $i=1, \ldots, l$. Moreover, if $s \notin\left\{z_{1}, \ldots, z_{l}\right\}$ is a root of $g(z)$ with the multiplicity $j$, then $s$ is also a root of $h(z)$ with the multiplicity greater or equal to $j$. All these conditions imply that we can write

$$
g(z)=-\alpha p(z) \tilde{g}(z), \quad h(z)=\alpha p(z) \tilde{h}(z),
$$

where $p(z), \tilde{g}(z)$ and $\tilde{h}(z)$ are monic polynomials; $p$ is of degree $k-l, \alpha \in \mathbb{C}^{*}$ and

$$
\tilde{g}(z)=\prod_{i=1}^{l}\left(z-z_{i}\right)
$$

Hence

and then

$$
\omega=-\frac{\tilde{h}(z)}{\tilde{g}(z)} \mathrm{d} z
$$

$$
\frac{1}{\Lambda_{i}}=\frac{h\left(z_{i}\right)}{g^{\prime}\left(z_{i}\right)}=-\frac{p\left(z_{i}\right) \tilde{h}\left(z_{i}\right)}{p^{\prime}\left(z_{i}\right) \tilde{g}\left(z_{i}\right)+p\left(z_{i}\right) \tilde{g}^{\prime}\left(z_{i}\right)}=-\frac{\tilde{h}\left(z_{i}\right)}{\tilde{g}^{\prime}\left(z_{i}\right)}=\operatorname{res}\left(\omega, z_{i}\right),
$$

for $i=1, \ldots, l$. Moreover, it is easy to see that $\operatorname{res}(\omega, \infty)=1$. This finishes the proof. 
REMARK 6.3 If $\boldsymbol{F}$ satisfies assumption A.1 and does not satisfy A.2 we still can do something. In such situation we have

$$
g(z)=-\alpha p(z) \tilde{g}(z), \quad p(z)=\prod_{i=1}^{s}\left(z-s_{i}\right)^{n_{i}}, \quad \tilde{g}(z)=\prod_{i=1}^{l}\left(z-z_{i}\right),
$$

and

$$
h(z)=\alpha q(z) \tilde{h}(z), \quad q(z)=\prod_{i=1}^{s}\left(z-s_{i}\right)^{m_{i}},
$$

where

$$
l+\sum_{i=1}^{s} n_{i}=k, \quad \operatorname{deg} \tilde{h}+\sum_{i=1}^{s} m_{i}=k-1,
$$

$s_{i} \notin\left\{z_{1}, \ldots, z_{l}\right\}$ and $\tilde{h}\left(s_{i}\right) \neq 0$ for $i=1, \ldots, s$. Now

$$
\omega=-\frac{\tilde{h}(z)}{\tilde{g}(z) \prod_{i=1}^{s}\left(z-s_{i}\right)^{n_{i}-m_{i}}} \mathrm{~d} z
$$

and the global residue theorem gives

$$
\sum_{i=1}^{l} \frac{1}{\Lambda_{i}}+\sum_{j=1}^{s} \operatorname{res}\left(\omega, s_{j}\right)=-1
$$

We notice that if $n_{i}>m_{i}$, then, generally, residue $\operatorname{res}\left(\omega, s_{i}\right) \neq 0$ and it depends on coefficients of polynomials $F_{1}$ and $F_{2}$.

\subsection{Non-maximal number of Darboux points and multiple Darboux points}

If a force $\boldsymbol{F}=\left(F_{1}, F_{2}\right)$ does not have the maximal number of Darboux points, then polynomials $F_{1}$ and $F_{2}$ have a common factor $P \in \mathbb{C}[\boldsymbol{q}] \backslash \mathbb{C}$, i.e., $F_{i}=P \widetilde{F}_{i}$, where $\widetilde{F}_{i} \in \mathbb{C}[\boldsymbol{q}]$ for $i=1,2$. In such cases it is possible to find a particular solution which does not correspond to a Darboux point. For example, if $P=q_{2}$, then $q_{1}(t)=\gamma t, q_{2}(t)=0$ is a particular solution. Amazingly, we can use such simple solutions and methods of differential Galois theory for proving the non-integrability. First we show a very simple fact.

Proposition 6.2. Let $\boldsymbol{F}(\boldsymbol{q})=\left(\alpha_{1} q_{1}+\alpha_{2} q_{2}\right)\left(\widehat{F}_{1}(\boldsymbol{q}), \widehat{F}_{2}(\boldsymbol{q})\right)$ where $\left|\alpha_{1}\right|+\left|\alpha_{2}\right| \neq \underset{\sim}{0}$, and $\widehat{F}_{1}, \widehat{F}_{2} \in \mathbb{C}[\boldsymbol{q}]$. Then there exists $A \in \mathrm{GL}(2, \mathbb{C})$, such that $A \boldsymbol{F}\left(A^{-1} \boldsymbol{q}\right)=q_{2}\left(\widetilde{F}_{1}(\boldsymbol{q}), \widetilde{F}_{2}(\boldsymbol{q})\right)$, where $\widetilde{F}_{i} \in \mathbb{C}[\boldsymbol{q}]$ for $i=1,2$.

Proof. Matrix $A$ given by

$$
A=\left[\begin{array}{cc}
\alpha_{2}^{\star} & -\alpha_{1}^{\star} \\
\alpha_{1} & \alpha_{2}
\end{array}\right], \quad \operatorname{det} A=\left|\alpha_{2}\right|^{2}+\left|\alpha_{2}\right|^{2},
$$

is non-singular and satisfies the desired condition. Here asterisk denotes the complex conjugation. 
Hence, if a force $\boldsymbol{F}=\left(F_{1}, F_{2}\right)$ where $\operatorname{deg} F_{i}=k-1$, has less than $k$ Darboux points, then we can always assume that $F_{1}$ and $F_{2}$ have a common factor $q_{2}$. We show that under a certain genericity condition the Newton equations with such force are not integrable in the Jacobi sense.

Theorem 6.2. Assume that $\widetilde{F}_{1}, \widetilde{F}_{2} \in \mathbb{C}[\boldsymbol{q}]$ are homogeneous polynomials of degree $k-2$. If $\operatorname{deg}_{q_{1}} \tilde{F}_{2}=k-2$, then system

$$
\dot{q}_{1}=p_{1}, \quad \dot{p}_{1}=-q_{2} \widetilde{F}_{1}\left(q_{1}, q_{2}\right), \quad \dot{q}_{2}=p_{2}, \quad \dot{p}_{2}=-q_{2} \widetilde{F}_{2}\left(q_{1}, q_{2}\right),
$$

does not admit two functionally independent polynomial first integrals.

Proof. System (6.24) possesses the following particular solution

$$
q_{1}(t)=\gamma t+\beta, \quad p_{1}(t)=\gamma, \quad q_{2}(t)=p_{2}(t)=0 .
$$

Variational equations along this solution take the form

$$
\left(\begin{array}{c}
\dot{Q}_{1} \\
\dot{P}_{1} \\
\dot{Q}_{2} \\
\dot{P}_{2}
\end{array}\right)=\left(\begin{array}{cccc}
0 & 1 & 0 & 0 \\
-\widetilde{F}_{1}\left(q_{1}(t)\right. & 0 & 0 & 0 \\
0 & 0 & 0 & 1 \\
0 & 0 & -\widetilde{F}_{2}\left(q_{1}(t), 0\right) & 0
\end{array}\right)\left(\begin{array}{c}
Q_{1} \\
P_{1} \\
Q_{2} \\
P_{2}
\end{array}\right)
$$

We have

$$
\tilde{F}_{1}\left(q_{1}, 0\right)=\alpha_{1} q_{1}^{k-2} \text { and } \quad \tilde{F}_{2}\left(q_{1}(t), 0\right)=\alpha_{2} q_{1}^{k-2},
$$

where $\alpha_{2} \neq 0$, as, by assumption $\operatorname{deg}_{q_{1}} \tilde{F}_{2}\left(q_{1}, 0\right)=k-2$. We choose constants of integration $\beta=0$ and $\gamma$ in such a way that $\alpha_{2} \gamma^{k-2}=-1$. Then the variational equations take the form

$$
\dot{Q}_{1}=P_{1}, \quad \dot{P}_{1}=\alpha t^{m} Q_{2}, \quad \dot{Q}_{2}=P_{2}, \quad \dot{P}_{2}=t^{m} Q_{2}, \quad \alpha \in \mathbb{C}, \quad m=k-2>0 .
$$

First we show that the differential Galois group $G$ of the above variational equations is a direct product $\{E\} \times \mathrm{SL}(2, \mathbb{C})$, where $E$ is the $2 \times 2$ identity matrix. To this end, we notice that $Q_{2}$ satisfies the following equation

$$
\ddot{Q}_{2}=t^{m} Q_{2} .
$$

Let $K \supset \mathbb{C}(t)$ be the Picard-Vessiot extention for this equation, i.e., $K$ is the smallest differential field containing two linearly independent solutions of (6.27). Then all solutions of (6.26) belong to $K^{4}$. It is clear for $\alpha=0$. If $\alpha \neq 0$, then $\ddot{Q}_{1}-\alpha \ddot{Q}_{2}=0$, and so $Q_{1}(t)=$ $\alpha Q_{2}(t)+a t+b$, for some $a, b \in \mathbb{C}$. So, as we claimed $\left(Q_{1}(t), \dot{Q}_{1}(t), Q_{2}(t), \dot{Q}_{2}(t)\right) \in K^{4}$.

Now we can determine the differential Galois group of variational equations (6.26). For this purpose we have to know the differential Galois $\widetilde{G}$ group of equation (6.27). In 22] it was shown that $\widetilde{G}=\mathrm{SL}(2, \mathbb{C})$ for an arbitrary $m>0$. Let $w_{1}$ and $w_{2}$ denote two linearly 
independent solutions of (6.27). Then the fundamental matrix of (6.26) has the following form

$$
X=\left[\begin{array}{cccc}
1 & t & \alpha w_{1} & \alpha w_{2} \\
0 & 1 & \alpha \dot{w}_{1} & \alpha \dot{w}_{2} \\
0 & 0 & w_{1} & w_{2} \\
0 & 0 & \dot{w}_{1} & \dot{w}_{2}
\end{array}\right]
$$

Now, if for $g \in \widetilde{G}$ we have

$$
g\left(w_{i}\right)=\sigma_{1, i} w_{1}+\sigma_{2, i} w_{2} \quad \text { for } \quad i=1,2,
$$

then $\Sigma=\left[\sigma_{i, j}\right] \in \mathrm{SL}(2, \mathbb{C})$, and

$$
g(X)=X S, \quad S=\left[\begin{array}{cc}
E & 0 \\
0 & \Sigma
\end{array}\right]
$$

where $E$ is the $2 \times 2$ identity matrix. Hence the differential Galois group $G$ of system (6.26) is a direct product $\{E\} \times \mathrm{SL}(2, \mathbb{C})$.

Let us notice that we cannot finish our proof at this point invoking Lemma 2.1. In fact, the differential Galois group $G$ has two independent invariants $Q_{1}$ and $P_{1}$. Thus we proceed in the following way. From the proof of Lemma 4.2 it follows that if $R \in \mathbb{C}\left(Q_{1}, P_{1}, Q_{2}, P_{2}\right)$ is an invariant of $G$, then $R \in \mathbb{C}\left(Q_{1}, P_{1}\right)$. This implies that if $I \in \mathbb{C}(t)\left(Q_{1}, P_{1}, Q_{2}, P_{2}\right)$ is a first integral of variational equations (6.26), then $I \in \mathbb{C}(t)\left(Q_{1}, P_{1}\right)$.

Let us assume that $P \in \mathbb{C}\left[q_{1}, p_{1}, q_{2}, p_{2}\right] \backslash \mathbb{C}$ is a first integral of (6.24). We can write it in the following form

$$
P=\sum_{0 \leq i+j \leq m} P_{i, j}\left(q_{1}, p_{1}\right) q_{2}^{i} p_{2}^{j} .
$$

The leading term $p \in C[t]\left(Q_{1}, P_{1}, Q_{2}, P_{2}\right)$ of $P$ is the lowest degree term of

$$
P\left(\gamma t+Q_{1}, \gamma+P_{1}, Q_{2}, P_{2}\right)=\sum_{0 \leq i+j \leq m} P_{i, j}\left(\gamma t+Q_{1}, \gamma+P_{1}\right) Q_{2}^{i} P_{2}^{j},
$$

and it is a first integral of variational equations (6.26). We have already shown that the integral $p$ must belong to $C[t]\left(Q_{1}, P_{1}\right)$. Hence $p$ is the lowest order term of $P_{0,0}\left(\gamma t+Q_{1}, \gamma+\right.$ $\left.P_{1}\right)$.

We show that $P_{0,0} \in \mathbb{C}\left[p_{1}\right]$. Inserting expansion (6.31) into equation

$$
p_{1} \frac{\partial P}{\partial q_{1}}+p_{2} \frac{\partial P}{\partial q_{2}}-q_{2} \widetilde{F}_{1}\left(q_{1}, q_{2}\right) \frac{\partial P}{\partial p_{1}}-q_{2} \widetilde{F}_{2}\left(q_{1}, q_{2}\right) \frac{\partial P}{\partial p_{2}}=0,
$$

obtained from the condition $\dot{P}=0$, and taking terms of degree zero with respect to $\left(q_{2}, p_{2}\right)$, we obtain

$$
p_{1} \frac{\partial P_{0,0}}{\partial q_{1}}=0,
$$

and hence $P_{0,0} \in \mathbb{C}\left[p_{1}\right]$, as we claimed. 
Now, assume that system (6.24) admits two independent polynomial first integrals $P$ and $Q$. We can assume that their leading terms $p, q \in C[t]\left(Q_{1}, P_{1}, Q_{2}, P_{2}\right)$ are independent. But we know that $p, q \in C[t]\left(Q_{1}, P_{1}\right)$, and thus, $p$ and $q$ are the lowest degree terms of $P_{0,0}\left(\gamma t+Q_{1}, \gamma+P_{1}\right)$ and $Q_{0,0}\left(\gamma t+Q_{1}, \gamma+P_{1}\right)$. However, we have already shown that $P_{0,0}$ and $Q_{0,0}$ depend only on $p_{1}$, so $p$ and $q$ depend only on $P_{1}$, and thus they are functionally dependent. A contradiction finishes the proof.

REMARK 6.4 If in the above lemma we assume that $\operatorname{deg}_{q_{1}} F_{1}=\operatorname{deg}_{q_{1}} F_{2}=k-2$, then $\alpha$ in variational equations (6.26) is not zero, and we can modify the final step of the proof. Let $P \in \mathbb{C}(t)\left(Q_{1}, P_{1}\right)$ be a first integral of variational equations (6.26). Then the condition $\dot{P}=0$ yields

$$
\frac{\partial P}{\partial t}+P_{1} \frac{\partial P}{\partial Q_{1}}+\alpha t^{m} Q_{2} \frac{\partial P}{\partial P_{1}}=0
$$

and thus $\frac{\partial P}{\partial P_{1}}=0$. This implies that the variational equations admit at most one rational first integral, and thus the considered system (6.24) is not integrable in the Jacobi sense with two rational first integrals.

REMARK 6.5 Let us assume that $\boldsymbol{F}(\boldsymbol{q})=\left(\alpha_{1} q_{1}+\alpha_{2} q_{2}\right)\left(\widehat{F}_{1}(\boldsymbol{q}), \widehat{F}_{2}(\boldsymbol{q})\right)$. Without loss of generality we can assume that $\alpha_{2} \neq 0$. If $\alpha_{2}=0$, we just renumber the coordinates. By Proposition 6.2 there exists a non-singular matrix $A$ such that

$$
\boldsymbol{F}_{A}(\boldsymbol{q}):=A \boldsymbol{F}\left(A^{-1} \boldsymbol{q}\right)=q_{2}\left(\widetilde{F}_{1}(\boldsymbol{q}), \widetilde{F}_{2}(\boldsymbol{q})\right) .
$$

Assumption $\operatorname{deg}_{q_{1}} \widetilde{F}_{2}=k-2$ in Theorem 6.2 means that $\widetilde{F}_{2}\left(q_{1}, 0\right) \neq 0$. We have

$$
\widetilde{F}_{2}\left(q_{1}, q_{2}\right)=\alpha_{1} \widehat{F}_{1}\left(\alpha_{2} q_{1}+\alpha_{1}^{\star} q_{2},-\alpha_{1} q_{1}+\alpha_{2}^{\star} q_{2}\right)+\alpha_{2} \widehat{F}_{2}\left(\alpha_{2} q_{1}+\alpha_{1}^{\star} q_{2},-\alpha_{1} q_{1}+\alpha_{2}^{\star} q_{2}\right),
$$

and thus

$$
\widetilde{F}_{2}\left(q_{1}, 0\right)=\alpha_{2}^{k-1} q_{1}^{k-2}\left[\alpha_{1} \widehat{F}_{1}\left(\alpha_{2},-\alpha_{1}\right)+\alpha_{2} \widehat{F}_{2}\left(\alpha_{2},-\alpha_{1}\right)\right] .
$$

The above shows that if the polynomial

$$
\widehat{G}(\boldsymbol{q}):=q_{1} \widehat{F}_{2}(\boldsymbol{q})-q_{2} \widehat{F}_{1}(\boldsymbol{q}),
$$

is divisible by $\left(\alpha_{1} q_{1}+\alpha_{2} q_{2}\right)$, then $\widetilde{F}_{2}\left(q_{1}, 0\right)=0$. On the other hand, if $\widetilde{F}_{2}\left(q_{1}, 0\right)=0$, then from (6.35) it follows that

$$
\hat{g}(\alpha):=\widehat{F}_{2}(1, \alpha)-\alpha \widehat{F}_{1}(1, \alpha)=\hat{f}_{2}(\alpha)-\alpha \hat{f}_{1}(\alpha)=0, \quad \alpha=-\frac{\alpha_{1}}{\alpha_{2}} .
$$

Thus $\alpha$ is a root of polynomial $\hat{g} \in \mathbb{C}[z]$, and this is equivalent to the fact that $\left(\alpha_{1} q_{1}+\alpha_{2} q_{2}\right)$ is a factor of $\widehat{G}(\boldsymbol{q})$.

It can happen that the considered force does not have Darboux points. Such forces are characterized in the following theorem. 
Theorem 6.3. Assume that a force $\boldsymbol{F}=\left(F_{1}, F_{2}\right), \operatorname{deg} F_{i}=k-1$, does not have a Darboux point. Then

$$
F_{1}=\prod_{i=1}^{k-1}\left(q_{2}-z_{i} q_{1}\right), \quad F_{2}=\frac{1}{q_{1}}\left[q_{2} F_{1}-\prod_{i=1}^{p}\left(q_{2}-z_{i} q_{1}\right)^{n_{i}}\right],
$$

where $1 \leq p \leq k-1, z_{i} \in \mathbb{C}$, and $z_{1}, \ldots, z_{p}$ are pairwise different; $n_{i} \in \mathbb{N}$ and $n_{1}+\cdots+n_{p}=$ $k$. Moreover, if there exist $1 \leq j \leq p$ such that $n_{j}=1$, then the Newton equations with force $\boldsymbol{F}$ do not admit two functionally independent polynomial first integrals.

Let us note that in spite of the fraction in the expression on $F_{2}$ the force $\boldsymbol{F}$ is polynomial, see the proof below.

Proof. Without loss of generality we can assume that $\operatorname{deg}_{q_{2}} F_{1}=k-1$. Then for polynomials $h, g \in \mathbb{C}[z]$ defined by (6.5) we have $\operatorname{deg} g=k$ and $\operatorname{deg} h=k-1$. Assumption that $\boldsymbol{F}$ have no Darboux point implies that all roots of $g$ are also roots of $h$. So $g$ has at least one multiple root and we can write it in the following form

$$
g(z)=\alpha \prod_{i=1}^{p}\left(z-z_{i}\right)^{n_{i}}, \quad n_{i} \in \mathbb{N}, \quad \sum_{i=1}^{p} n_{i}=k, \quad \alpha \in \mathbb{C}^{\star},
$$

where $1 \leq p \leq k-1$ and $z_{1}, \ldots, z_{p}$ are pairwise different roots of $h$. Let $z_{p+1}, \ldots, z_{k-1}$ be the remaining (not necessarily different) roots of $h$. Then we can write

$$
h(z)=\beta \prod_{i=1}^{k-1}\left(z-z_{i}\right) .
$$

Thus $f_{1}(z)=h(z)$, and

$$
f_{2}(z)=g(z)+z h(z)=\alpha \prod_{i=1}^{p}\left(z-z_{i}\right)^{n_{i}}+\beta z \prod_{i=1}^{k-1}\left(z-z_{i}\right) .
$$

But $\operatorname{deg} f_{2}(z)<k$, thus $\alpha=-\beta$ and we can put $\beta=1$. Now, as $F_{i}\left(q_{1}, q_{2}\right)=q_{1}^{k-1} f_{i}\left(q_{2} / q_{1}\right)$ for $i=1,2$, we obtain (6.37) and this finishes the first statement of our theorem.

To proof the second statement let us assume that $n_{1}=1$. Then we can write

$$
F_{i}=\left(q_{2}-z_{1} q_{1}\right) \widehat{F}_{i} \quad \text { for } \quad i=1,2,
$$

where

$$
\widehat{F}_{1}=\prod_{i=2}^{k-1}\left(q_{2}-z_{i} q_{1}\right) \quad \widehat{F}_{2}=\frac{1}{q_{1}}\left[q_{2} \widehat{F}_{1}-\prod_{i=2}^{p}\left(q_{2}-z_{i} q_{1}\right)^{n_{i}}\right],
$$

Let us calculate polynomial $\hat{g}(z)$ as in Remark 6.5. We have

$$
\hat{g}(z)=\widehat{G}(1, z)=\widehat{F}_{2}(1, z)-z \widehat{F}_{1}(1, z)=-\prod_{i=2}^{p}\left(z-z_{i}\right)^{n_{i}}
$$

so $g\left(z_{1}\right) \neq 0$. Thus, as we explained in Remark 6.5. one can apply Theorem 6.2 to the considered case and this finishes the proof. 
Let us notice that if the assumptions of the above theorem are satisfied, then the considered Newton's system still can possess a single first integral. Let

$$
F_{1}\left(q_{1}, q_{2}\right)=\prod_{i=1}^{k-1}\left(q_{2}-z_{i} q_{1}\right) \quad \text { and } \quad F_{2}\left(q_{1}, q_{2}\right)=z_{j} F_{1}\left(q_{1}, q_{2}\right)
$$

where $1 \leq j \leq k-1$ and $z_{1}, \ldots, z_{k-1}$ are pairwise different. This force has the form (6.37) with $p=k-1, n_{i}=1$ for $i \neq j$ and $n_{j}=2$. However, the respective Newton system possesses a first integral

$$
I=z_{j} p_{1}-p_{2} .
$$

If in (6.37) all $n_{i}$ are greater than one, then the system can be integrable. We give such examples in the next section.

\subsection{Infinitely many Darboux points}

If

$$
q_{1} F_{2}\left(q_{1}, q_{2}\right)=q_{2} F_{1}\left(q_{1}, q_{2}\right),
$$

then force $\boldsymbol{F}=\left(F_{1}, F_{2}\right)$ has infinitely many Darboux points. At each of these points the Kovalevskaya exponents are $\Lambda_{1}=0$ and $\Lambda_{2}=k-1$.. Thus the Morales-Ramis theory does not give any obstructions for the integrability.

It is easy to show that condition (6.41) is satisfied if and only if

$$
F_{1}=\sum_{i=0}^{k-2} f_{i} q_{1}^{k-1-i} q_{2}^{i} \quad \text { and } \quad F_{2}=\sum_{i=0}^{k-2} f_{i} q_{1}^{k-2-i} q_{2}^{i+1} .
$$

The Newton system with a force satisfying (6.41) possesses one first integral

$$
I=q_{1} p_{2}-q_{2} p_{1} .
$$

For $k=3$ and $k=4$ the Newton equations with the above form (6.42) of forces are integrable with an additional first integral quadratic in the momenta

$$
\begin{aligned}
& I_{2}=3\left(f_{0} p_{1}+f_{1} p_{2}\right)^{2}+2\left(f_{0} q_{1}+f_{1} q_{2}\right)^{3}, \\
& I_{2}=2\left(f_{0} p_{1}^{2}+f_{1} p_{1} p_{2}+f_{2} p_{2}^{2}\right)+\left(f_{0} q_{1}^{2}+f_{1} q_{1} q_{2}+f_{2} q_{2}^{2}\right)^{2},
\end{aligned}
$$

respectively. But for $k>4$, it seems that the Newton equations with forces given by (6.42) are not integrable in a generic case, nevertheless, they admit an additional first integral for some special values of $f_{i}$. For example, if

$$
F_{1}=q_{1}\left(f_{0} q_{1}+f_{1} q_{2}\right)^{k-2} \text { and } F_{2}=q_{2}\left(f_{0} q_{1}+f_{1} q_{2}\right)^{k-2},
$$

then the additional first integral has the following form

$$
I=k\left(f_{0} p_{1}+f_{1} p_{2}\right)^{2}+2\left(f_{0} q_{1}+f_{1} q_{2}\right)^{k} .
$$

We do not know how to investigate systematically the integrability of the Newton equations in this case. It seems that we can apply only a direct method. 
Lemma 6.1. Assume that force $\boldsymbol{F}=\left(F_{1}, F_{2}\right)$ admits infinitely many Darboux points and the corresponding Newton equations have a polynomial first integral which is a quadratic form with constant coefficients with respect to the momenta. Then these equations are equivalent to an integrable Hamiltonian system with the radial potential

$$
H=\frac{1}{2}\left(p_{1}^{2}+p_{2}^{2}\right)+\frac{1}{k}\left(q_{1}^{2}+q_{2}^{2}\right)^{\frac{k}{2}},
$$

for $k$ even, or to the non-Hamiltonian integrable system

$$
F_{1}=q_{1} q_{2}^{k-2}, \quad F_{2}=q_{2}^{k-1}, \quad I_{2}=\frac{1}{2} p_{2}^{2}+\frac{1}{k} q_{2}^{k},
$$

for any $k$.

Proof. Without loss of the generality one can postulate the following form of the first integral quadratic in the momenta

$$
I=\alpha p_{1}^{2}+p_{2}^{2}+\sum_{i=0}^{k} b_{k} q_{1}^{k-i} q_{2}^{i} .
$$

The case when the quadratic form of momenta is nonsingular corresponds (after rescaling) to $\alpha=1$ and the case when this form is singular corresponds to $\alpha=0$. Condition $\dot{I}=0$ on solutions of (6.42) yields

$$
\begin{aligned}
& (k-i) b_{i}-2 \alpha f_{i}=0, \quad i=0, \ldots k-2, \quad b_{k-1}=0, \\
& b_{1}=0, \quad(i+2) b_{i+2}-2 f_{i}=0, \quad i=0, \ldots, k-2,
\end{aligned}
$$

and the detailed analysis of solutions of this linear system yields the desired result.

\section{Two degrees of freedom. Applications}

In this section we consider also Newton equations with two degrees of freedom but now we apply general theorems from the previous sections to general homogeneous forces of low degrees. The first aim is to show that our general results allow to perform the integrability analysis in a systematic and algorithmic way. Let us describe the main steps of this algorithm.

We fix $k>2$ and consider the Newton system with a homogeneous polynomial force $\boldsymbol{F}=\left(F_{1}, F_{2}\right)$ where $\operatorname{deg} F_{1}=\operatorname{deg} F_{2}=k-1$. The problem is to give a complete list of all $\boldsymbol{F}$ for which the corresponding Newton system is integrable in the Jacobi sense. As $\mathbb{C}$-linear space $\mathbb{C}_{k-1}[\boldsymbol{q}]$ of homogeneous polynomials in two variables of degree $k-1$ has the dimension $k$, the problem depends on $2 k$ parameters. We can reduce the dimension of the problem taking into account the action of the four dimensional group $\mathrm{GL}(2, \mathbb{C})$.

If for a given $k$ a complex number $\lambda$ belongs to an item in table (1.13) of Theorem 1.3, then we write $\lambda \in \mathcal{N}_{k}$. 
In the first step of the algorithm we have to find for $1 \leq l \leq k$ all solutions of equation

$$
\sum_{i=1}^{l} \frac{1}{\Lambda_{i}}=-1
$$

such that $\lambda_{i}=\Lambda_{i}+1 \in \mathcal{N}_{k}$ for $i=1, \ldots, l$. The set of such solutions we denote as in [22] by $\mathcal{I}_{k, l}$. Let us remark here that for small $k$ it can be determined easily with the help of a computer algebra program, however, for bigger $k$ it is a time demanding computational problem.

In the next step for each solution $\left(\Lambda_{1}, \ldots, \Lambda_{l}\right)$ obtained in the previous step we have to determine all non-equivalent forces $\boldsymbol{F}$ with exactly $l$ simple Darboux points, and such that at $i$-th point the non-trivial Kovalevskaya exponent is $\Lambda_{i}$, for $i=1, \ldots, l$. For small $k$ it can be done effectively, however, for bigger $k$ the problem is hard, because we have to solve a system of nonlinear polynomial equations of high degrees. The forces selected in this way satisfy all necessary integrability conditions of Theorem 1.3 but of course not all of them are integrable. To decide which of them are integrable, we have to use a theorem giving the stronger necessary integrability conditions than those contained in Theorem 1.3 . However, as far as we know, there is no such a theorem. In Appendix B we present a possible approach to this problem. It is a direct generalization of the method based on higher order variational equations developed in [24; 27] for Hamilton equations.

The above steps do not finish the procedure. We have to investigate separately all the cases when the force have lower than the maximal number of Darboux points with a multiple Darboux point or does not have such a point at all, because for those cases we cannot apply Theorem 1.3. but, nevertheless, we can use Theorem 6.2. Moreover, in all considered cases we have to distinguish all instances when the Newton system is Hamiltonian.

Below, in a case when we cannot apply any theorem to check the integrability, we use several different algorithms for finding a polynomial first integrals of a given degree. We do not describe details of these computations and below we call such a search a direct method.

\subsection{Case $k=3$}

Here we investigate the Newton equations with a homogeneous force $\boldsymbol{F}=\left(F_{1}, F_{2}\right)$ of degree two

$$
F_{1}=\frac{a_{11}}{2} q_{1}^{2}+a_{12} q_{1} q_{2}+\frac{a_{13}}{2} q_{2}^{2}, \quad F_{2}=\frac{a_{21}}{2} q_{1}^{2}+a_{22} q_{1} q_{2}+\frac{a_{23}}{2} q_{2}^{2} .
$$

Let us assume that $\boldsymbol{F}$ possesses at least one Darboux point. Then it can be placed at the infinity, so $F_{1}\left(0, q_{2}\right)=0$, and thus in (7.1) we put $a_{13}=0$. Additionally, by a linear change of variables $q_{1}=a Q_{1}$ and $q_{2}=b Q_{1}+c Q_{2}$ where $a c \neq 0$ one can obtain $a_{22}=0$.

As explained in Appendix A, for $k=3$ the Newton equations are almost always Hamiltonian. After the above simplifications the Newton system is not Hamiltonian only 
when

$$
\begin{array}{llll}
a_{12}=0 & \text { and } & a_{21} a_{23} \neq 0, & \text { or } \\
a_{21}=0 & \text { and } & a_{12}\left(a_{23}-a_{12}\right) \neq 0 . &
\end{array}
$$

Hamiltonian systems with homogeneous potentials of degree 3 were fully analyzed in [20], so we consider here only non-Hamiltonian cases. At first, let us assume that conditions (7.2) are satisfied. Then, as $a_{21} \neq 0$ and $a_{23} \neq 0$, we can make a rescaling which gives $a_{21}=-1$ and $a_{23}=1$. Hence we have to consider the following one parameter family of Newton's equations

$$
\begin{aligned}
& \ddot{q}_{1}=-\frac{1}{2} \alpha q_{1}^{2}, \quad \alpha \in \mathbb{C}, \\
& \ddot{q}_{2}=\frac{1}{2} q_{1}^{2}-\frac{1}{2} q_{2}^{2} .
\end{aligned}
$$

This system has one first integral

$$
I=3 p_{1}^{2}+\alpha q_{1}^{3},
$$

and for the complete integrability one additional first integral is necessary.

For (7.4) polynomials $g(z)$ and $h(z)$, see definitions (6.5), have the forms

$$
g(z)=\frac{1}{2}\left(z^{2}+\alpha z-1\right), \quad h(z)=\frac{1}{2} \alpha .
$$

Thus

$$
z_{1,2}=\frac{\alpha \pm \sqrt{\alpha^{2}+4}}{2},
$$

are two Darboux points provided $\alpha \neq 0$. If $\alpha \neq \pm 2 \mathrm{i}$, then $z_{1} \neq z_{2}$. The non-trivial Kovalevskaya exponents at these points are

$$
\Lambda_{1,2}= \pm \sqrt{1+\frac{4}{\alpha^{2}}}
$$

Now, if system (7.4) is integrable, then $\Lambda_{1}$ and $\Lambda_{2}$ belong to table (1.13) of Theorem 1.3. It is easy to notice that there are only two possibilities: either $\Lambda_{1}=\Lambda_{2}=0$, or $\Lambda_{1}=-\Lambda_{2}=1$. But for $\Lambda_{i}$ given by (7.6) the second case is impossible. The first case gives $\alpha= \pm 2 \mathrm{i}$.

Summarizing, we have to investigate equations (7.4) only when $\alpha= \pm 2 \mathrm{i}$ and $\alpha=0$.

Assume that $\alpha=0$. We introduce new coordinates

$$
Q_{1}=q_{1}+q_{2}, \quad Q_{2}=q_{1}-q_{2} .
$$

In these coordinates equations (7.4) read

$$
\ddot{Q}_{1}=\frac{1}{2} Q_{1} Q_{2}, \quad \ddot{Q}_{2}=-\frac{1}{2} Q_{1} Q_{2} .
$$


Applying Theorem 6.2 to this system, we conclude that for $\alpha=0$ equations (7.4) are not integrable in the Jacobi sense with polynomial first integrals.

If $\alpha= \pm 2 \mathrm{i}$, then the considered force has a double Darboux point. At this Darboux point the non-trivial Kovalevskaya exponent is $\Lambda=0$. Making the following change of variables

$$
Q_{1}=-\frac{\alpha}{4} q_{1}+\frac{1}{2} q_{2}, \quad Q_{2}=\frac{\alpha}{2} q_{1},
$$

we transform system (7.4) for the prescribed values of $\alpha$ into the following one

$$
\ddot{Q}_{1}=-Q_{1}\left(Q_{1}+Q_{2}\right), \quad \ddot{Q}_{2}=-Q_{2}^{2},
$$

and now the double Darboux point is at infinity. Let us consider a phase curve $\Gamma_{e}, e \neq 0$, corresponding to this Darboux point. It is given by

$$
e=\frac{1}{2} \dot{Q}_{2}^{2}+\frac{1}{3} Q_{2}^{3}, \quad Q_{1}=\dot{Q}_{1}=0 .
$$

The particular solution corresponding to this phase curve is given by $Q_{2}(t)=-6 \wp\left(t, g_{2}, g_{3}\right)$, where invariants of the Weierstrass function are $g_{2}=0$ and $g_{3}=-e / 18$. Variational equations along this particular solution have the following form

$$
\ddot{\xi}_{1}=6 \wp\left(t, g_{2}, g_{3}\right) \xi_{1}, \quad \ddot{\xi}_{2}=12 \wp\left(t, g_{2}, g_{3}\right) \xi_{2},
$$

i.e., they are a direct product of two Lamé equation of the Lamé-Hermite type. Assuming our Conjecture B.1 we can apply the higher order variational equations for testing the integrability in the considered case. Calculations show that a logarithmic term appears in the second order variational equations, so the system is not integrable.

If condition (7.3) is satisfied, then either we obtain a system equivalent to (7.4), or to the following one

$$
\ddot{q}_{1}=-\lambda q_{1} q_{2}, \quad \ddot{q}_{2}=-q_{2}^{2}, \quad \lambda \in \mathbb{C}^{\star} .
$$

If $\lambda$ belongs to one of iteme in table (1.13) (with $k=3$ ), then the necessary conditions of Theorem 1.3 are satisfied. It seems that for all these values the force admits two or three independent polynomial first integrals. A family of forces with the above properties can be find for an arbitrary $k>2$. We discuss these families in the last subsection of this section.

At the beginning, we assumed that the force admits at least one Darboux point. Hence now, we consider all cases when there is no a Darboux point. By Theorem 6.3 we can assume that the force has the form given by (6.37) with $k=3$. If in (6.37) $p=2$, then $n_{1}=1$ or $n_{2}=1$ and the system is not integrable. When $p=1, n_{1}=3$ the force has the following components

$$
F_{1}=\left(q_{2}-z_{1} q_{1}\right)\left(q_{2}-z_{2} q_{1}\right), \quad F_{2}=\left(q_{2}-z_{1} q_{1}\right)\left(q_{2}\left(2 z_{1}-z_{2}\right)-z_{1}^{2} q_{1}\right) .
$$

Let $Q_{1}=q_{1}$ and $Q_{2}=q_{2}-z_{1} q_{1}$. Then in new variables the Newton equations with the above force read

$$
\ddot{Q}_{1}=Q_{2}\left(Q_{2}-\alpha Q_{1}\right), \quad \ddot{Q}_{2}=-\alpha Q_{2}^{2}, \quad \alpha \in \mathbb{C},
$$


and have one first integral

$$
I=\frac{1}{2} P_{2}^{2}+\frac{1}{3} \alpha Q_{2}^{3}, \quad P_{2}=\dot{Q}_{2} .
$$

If $\alpha=0$, then system (17.12) is a superintegrable Hamiltonian system with the Hamilton function

$$
H=P_{1} P_{2}-\frac{1}{3} Q_{2}^{3},
$$

and two additional first integrals

$$
I_{1}=P_{2}, \quad I_{2}=4 P_{2}\left(Q_{1} P_{2}-Q_{2} P_{1}\right)+Q_{2}^{4} .
$$

If $\alpha \neq 0$, we can assume that $\alpha=1$. For this case, using a direct method, we checked that the system (7.12) does not admit a second polynomial first integral of degree lower than 12 with respect to the momenta.

\subsection{Case $k=4$}

To shorten the exposition, we omit proofs and many details of calculations in this subsection. At first, we recall from paper [22] that for $k=4$ set $\mathcal{I}_{4,4}$ of $\Lambda_{i}=\lambda_{i}-1$ with $\lambda_{i}$ satisfying assumptions of Theorem 1.3 has elements listed in Table 1 , and $\mathcal{I}_{4, l}=\emptyset$ for $2 \leq l<4$.

\begin{tabular}{|c|c|}
\hline element & $\left\{\Lambda_{1}, \Lambda_{2}, \Lambda_{3}, \Lambda_{4}\right\}$ \\
\hline 1 & $\{-1,-1,2,2\}$ \\
2 & $\{-5 / 8,5,5,5\}$ \\
3 & $\{-5 / 8,2,20,20\}$ \\
4 & $\{-5 / 8,27 / 8,27 / 8,135\}$ \\
5 & $\{-5 / 8,2,14,35\}$ \\
\hline
\end{tabular}

Table 1: Elements of set $\mathcal{I}_{4,4}$.

\subsubsection{Four Darboux points}

If we assume that a homogeneous force $\boldsymbol{F}=\left(F_{1}, F_{2}\right)$ has four simple Darboux points, then, by a proper choice of coordinates, three of them can be located arbitrarily on the Riemann sphere. Thus, we can assume that one of them is at infinity, and two of them at $z=0$ and $z=1$, respectively. Under these assumptions we have

$$
\begin{aligned}
& F_{1}=a_{11} q_{1}^{3}+a_{12} q_{1}^{2} q_{2}+a_{13} q_{1} q_{2}^{2}, \\
& F_{2}=a_{21} q_{1}^{2} q_{2}+a_{22} q_{1} q_{2}^{2}+a_{23} q_{2}^{3},
\end{aligned}
$$

where

$$
a_{11}+a_{12}+a_{13}=a_{21}+a_{22}+a_{23}=1,
$$


and

$$
a_{11} a_{23} \neq 0 \text {, and } a_{11} \neq a_{21} \text {, and } a_{13} \neq a_{23}, \text { and } a_{11}-a_{21} \neq a_{13}-a_{23} .
$$

Now $z$-coordinates of Darboux points are

$$
z_{0}=0, \quad z_{1}=1, \quad z_{2}=\frac{a_{11}-a_{21}}{a_{13}-a_{23}}, \quad z_{3}=\infty .
$$

The respective non-trivial Kovalevskaya exponents at these points are the following

$$
\Lambda_{0}=\frac{a_{1}}{a_{11}}, \quad \Lambda_{1}=a_{3}-a_{1}, \quad \Lambda_{2}=\frac{\left(a_{1}-a_{3}\right) a_{1} a_{3}}{a_{11} a_{3}^{2}+a_{12} a_{1} a_{3}+a_{13} a_{1}^{2}}, \quad \Lambda_{3}=-\frac{a_{3}}{a_{13}+a_{3}},
$$

where

$$
a_{1}=a_{21}-a_{11}, \quad a_{2}=a_{22}-a_{12}, \quad a_{3}=a_{23}-a_{13} .
$$

Now we can take the admissible values of $\Lambda_{i}, i=1,2,3,4$ from Table 1 and reconstruct $F_{1}$ and $F_{2}$.

For $\left(\Lambda_{0}, \Lambda_{1}, \Lambda_{2}, \Lambda_{3}\right)=(-1,-1,2,2)$ we obtain

$$
\begin{aligned}
& F_{1}=\frac{q_{1}}{5}\left[-\left(2 a_{2}+1\right) q_{1}^{2}+5 a_{2} q_{1} q_{2}+3\left(2-a_{2}\right) q_{2}^{2}\right], \\
& F_{2}=\frac{q_{2}^{2}}{5}\left[\left(a_{2}+3\right) q_{1}+\left(2-a_{2}\right) q_{2}\right], \quad a_{2} \notin\left\{-\frac{1}{2}, 2\right\},
\end{aligned}
$$

for $\left(\Lambda_{0}, \Lambda_{1}, \Lambda_{2}, \Lambda_{3}\right)=(5,5,-5 / 8,5)$ :

$$
\begin{aligned}
& F_{1}=\frac{q_{1}}{5}\left[\left(a_{2}-7\right) q_{1}^{2}+5 a_{2} q_{1} q_{2}+6\left(2-a_{2}\right) q_{2}^{2}\right], \\
& F_{2}=\frac{q_{2}}{5}\left[6\left(a_{2}-7\right) q_{1}^{2}+5\left(9-a_{2}\right) q_{1} q_{2}+\left(2-a_{2}\right) q_{2}^{2}\right], \quad a_{2} \notin\{2,7\},
\end{aligned}
$$

for $\left(\Lambda_{0}, \Lambda_{1}, \Lambda_{2}, \Lambda_{3}\right)=(2,20,-5 / 8,20)$ :

$$
\begin{aligned}
& F_{1}=\frac{q_{1}}{11}\left[10\left(a_{2}-22\right) q_{1}^{2}+11 a_{2} q_{1} q_{2}+21\left(11-a_{2}\right) q_{2}^{2}\right], \\
& F_{2}=\frac{q_{2}}{11}\left[30\left(a_{2}-22\right) q_{1}^{2}+\left(660-29 a_{2}\right) q_{1} q_{2}+\left(11-a_{2}\right) q_{2}^{2}\right], \quad a_{2} \notin\{11,22\},
\end{aligned}
$$

for $\left(\Lambda_{0}, \Lambda_{1}, \Lambda_{2}, \Lambda_{3}\right)=(-5 / 8,27 / 8,27 / 8,135)$ :

$$
\begin{aligned}
& F_{1}=\frac{q_{1}}{220}\left[27\left(22-5 a_{2}\right) q_{1}^{2}+220 a_{2} q_{1} q_{2}-17\left(22+5 a_{2}\right) q_{2}^{2}\right], \\
& F_{2}=\frac{q_{2}}{1760}\left[81\left(22-5 a_{2}\right) q_{1}^{2}+410 a_{2} q_{1} q_{2}-\left(22+5 a_{2}\right) q_{2}^{2}\right], \quad a_{2} \notin\left\{-\frac{22}{5}, \frac{22}{5}\right\},
\end{aligned}
$$

and for $\left(\Lambda_{0}, \Lambda_{1}, \Lambda_{2}, \Lambda_{3}\right)=(2,35,-5 / 8,14)$ :

$$
\begin{aligned}
& F_{1}=\frac{q_{1}}{16}\left[7\left(2 a_{2}-77\right) q_{1}^{2}+16 a_{2} q_{1} q_{2}+15\left(37-2 a_{2}\right) q_{2}^{2}\right], \\
& F_{2}=\frac{q_{2}}{16}\left[21\left(2 a_{2}-77\right) q_{1}^{2}+4\left(399-10 a_{2}\right) q_{1} q_{2}+\left(37-2 a_{2}\right) q_{2}^{2}\right], \quad a_{2} \notin\left\{\frac{37}{2}, \frac{77}{2}\right\} .
\end{aligned}
$$


Permutations of allowable values of $\left(\Lambda_{0}, \Lambda_{1}, \Lambda_{2}, \Lambda_{3}\right)$ give equivalent forces in all the cases listed above.

As it has already been mentioned, there is no forces satisfying conditions A.1 and A.2 formulated before Theorem 6.1] with a smaller number of simple Darboux points.

For forces (7.14)-(7.18) the necessary integrability conditions formulated in Theorem 1.3 are satisfied. Thus, to check if they are integrable, we applied the method of higher order variational equations. Our calculations for forces (7.15) $-(7.18)$ show that the only integrable cases correspond to known Hamiltonian systems with homogeneous potentials. For a Newton equation with force (17.14) the variational equations do not reduce to the product of two Lamé equations and the application of higher order variational equations is an open problem.

We also checked whether the Newton equations with forces (7.14) $-(7.18)$ admit a single polynomial first integral for specific values of $a_{2}$. To this end, we applied a direct method, and we looked for first integrals which have degree lower than 8 with respect to the momenta. The results of these calculations are following.

Force (7.14) admits a polynomial first integral only for $a_{2}=-3$, but in this case the Newton equations are Hamiltonian and integrable.

Force (7.15) has always a polynomial first integral

$$
\begin{aligned}
I_{1} & =q_{1}\left(q_{1}-q_{2}\right) q_{2}\left[\left(a_{2}-7\right) q_{1}-\left(a_{2}-2\right) q_{2}\right]^{2}-5\left(a_{2}-7\right) q_{2} p_{1}^{2}-5\left(a_{2}-2\right) q_{1} p_{2}^{2} \\
& +5\left[\left(a_{2}-7\right) q_{1}+\left(a_{2}-2\right) q_{2}\right] p_{1} p_{2} .
\end{aligned}
$$

Furthermore, for $a_{2} \in\{-3,9 / 2,12\}$ the system is Hamiltonian and integrable.

For (7.16) if $a_{2}=0$ the system is Hamiltonian and integrable while for $a_{2}=\frac{33}{31}(21 \pm \sqrt{7})$ is Hamiltonian but non-integrable. Except these Hamiltonian cases only for $a_{2}=22$ force (7.16) admits a polynomial first integral of the form

$$
I=12 p_{2}^{4}+8 q_{1}\left(q_{2}^{7}+4 p_{2}^{2} q_{2}^{3}\right)-4 p_{2}\left(2 p_{1}+3 p_{2}\right) q_{2}^{4}+3 q_{2}^{8}-8 q_{1}^{2} q_{2}^{6} .
$$

Force (7.17) admits polynomial first integrals only for $a_{2}=\left\{0, \pm \frac{22}{25} \sqrt{\frac{4683}{43}}\right\}$ when the system is Hamiltonian and non-integrable.

Finally, force (7.17) admits a polynomial first integral only for $a_{2} \in\left\{0, \frac{3}{22}(285 \mp 4 \sqrt{86})\right\}$ when the system is Hamiltonian and non-integrable.

\subsubsection{Smaller number of simple Darboux points}

If the considered force admits fewer than four simple Darboux points, than we have to investigate all the cases when condition A.2 formulated before Theorem6.1] is not satisfied. It is easy to check that the case when $k=4$, and the system possesses three Darboux points is impossible.

Hence, we consider forces with two simple Darboux points. It can be shown that in this case the force is given by

$$
\begin{aligned}
& F_{1}=q_{1}\left(q_{1}-q_{2}\right)\left[\left(\lambda_{2}-1\right) q_{1}+\left(\lambda_{1}-1\right) \lambda_{2} q_{2}\right], \\
& F_{2}=q_{2}\left(q_{1}-q_{2}\right)\left[\left(\lambda_{1}-1\right) q_{2}+\left(\lambda_{2}-1\right) \lambda_{1} q_{1}\right] .
\end{aligned}
$$


provided that $\lambda_{1} \neq 1$ and $\lambda_{2} \neq 1$. If $\lambda_{1}=1$ or $\lambda_{2}=1$, then the above force has infinitely many Darboux points and, as we showed in Section 6.4 the corresponding Newton system is integrable. The above force satisfies the necessary conditions of Theorem 1.3 iff $\lambda_{1}$ and $\lambda_{2}$ take values from table (1.13). Let us notice that to derive (7.19) we have to use a generalized relation (6.23) as described in Remark 6.3.

Components of force (7.19) have a common factor $q_{1}-q_{2}$, so we can use Theorem 6.2. Applying Proposition 6.2 we easily transform the force to the form $F_{i}=q_{2} \widetilde{F}_{i}$, where $\widetilde{F}_{i}$ are homogeneous polynomials of degree 2 for $i=1,2$. It appears that $\operatorname{deg}_{q_{1}} \widetilde{F}_{2}=2$ iff $\lambda_{1} \neq \lambda_{2}$. Hence, by Theorem 6.2, the Newton system with force (7.19) is not integrable if $\lambda_{1} \neq \lambda_{2}$.

If $\lambda=\lambda_{1}=\lambda_{2} \neq 1$ is given by an item from table (1.13), then, in some cases, we were able to find one first integral. For example, if $\lambda=6$, then the first integral has the form

$$
I=q_{2} p_{1}^{2}+q_{1} p_{2}^{2}-\left(q_{1}+q_{2}\right) p_{1} p_{2}+5 q_{1} q_{2}\left(q_{2}-q_{1}\right)^{3} .
$$

Also when $\lambda_{1} \neq \lambda_{2}$ we can find one first integral. For example, for $\lambda_{1}=3 / 8$ and $\lambda_{2}=6$ the first integral reads

$$
I=p_{2}\left(q_{1} p_{2}-q_{2} p_{1}\right)-\frac{5}{8} q_{1} q_{2}^{2}\left(q_{1}-q_{2}\right)^{2} .
$$

Now we consider the case with only one Darboux point. It can be shown that in this case the force is given by

$$
\begin{aligned}
& F_{1}=\alpha q_{1} q_{2}\left(q_{1}+q_{2}\right), \quad F_{2}=q_{2}\left(q_{1}+q_{2}\right)\left[(1-\alpha) q_{1}+q_{2}\right], \quad \alpha \neq 1, \quad \text { or } \\
& F_{1}=\alpha q_{1}^{2} q_{2}, \quad F_{2}=q_{2}^{2}\left(q_{2}+\alpha q_{1}\right), \quad \text { or } \\
& F_{1}=\lambda q_{1} q_{2}^{2}, \quad F_{2}=q_{2}^{3} \text {. }
\end{aligned}
$$

By Theorem 6.2, the Newton system with force (7.20) is not integrable. For $\alpha=0$ the Newton equations with force (7.21) are Hamiltonian and integrable, while for $\alpha \neq 0$ they are also Hamiltonian, however nonintegrable, as it was proved in [22]. The case of force (7.22) is discussed in the last subsection of this section.

Assuming that Conjecture B.1 is valid, and applying the higher order variational equations method similarly as it was done for Hamiltonian systems in [20; 22], it is possible to prove the following.

Lemma 7.1. The only integrable Newton systems with $k=4$ possessing a multiple Darboux point are equivalent to the following one

$$
\ddot{q}_{1}=-q_{1}\left(a_{11} q_{1}^{2}+q_{2}^{2}\right), \quad \ddot{q}_{2}=-q_{2}\left(a_{11} q_{1}^{2}+q_{2}^{2}\right),
$$

with first integrals

$$
I_{1}=q_{1} p_{2}-q_{2} p_{1}, \quad I_{2}=\frac{1}{2}\left(a_{11} p_{1}^{2}+p_{2}^{2}\right)+\frac{1}{4}\left(a_{11} q_{1}^{2}+q_{2}^{2}\right)^{2} .
$$


Finally, let us consider the integrability problem for forces without a Darboux point. Then by Theorem 6.3 the only forces that can admit two first integrals are either of the following:

$$
\begin{array}{lll}
F_{1}=q_{2}\left[q_{2}\left(q_{1}+\alpha q_{2}\right)+q_{1}^{2}\right], & F_{2}=q_{2}^{2}\left(q_{1}+q_{2}\right), & \text { or } \\
F_{1}=0, & F_{2}=-q_{1} q_{2}^{2}, & \text { or } \\
F_{1}=q_{1}^{2} q_{2}, & F_{2}=-\alpha q_{1} q_{2}^{2}, & \text { or } \\
F_{1}=0, & F_{2}=q_{1}^{3}, & \text { or } \\
F_{1}=q_{2}^{2}\left(q_{1}+q_{2}\right), & F_{2}=q_{2}^{3} . &
\end{array}
$$

The force field (7.23) for any $\alpha$ possesses a first integral that is a nondegenerate quadratic form with respect to the momenta with constant coefficients, thus it is always Hamiltonian, however we found the second functionally independent first integral only for $\alpha=0$. Calculations were made up to degree 8 in the momenta. The force with components (7.24) has one first integral $I=p_{1}$ and there is no the second polynomial first integral up to degree 9 in the momenta. Newton system governed by (7.26) is integrable and Hamiltonian. The last force (7.27) is non-Hamiltonian with one first integral $I=2 p_{2}^{2}+q_{2}^{4}$ and the direct method does not yield the second polynomial first integral up to degree 10 in the momenta.

The most puzzling are forces (7.25). The application of the direct method up to degree 7 in the momenta yields one integrable Hamiltonian case for $\alpha=-1$, the another one integrable non-Hamiltonian for $\alpha=-11 / 6$ with two first integrals which are both of degree four in the momenta

$$
\begin{aligned}
& I_{1}=24 p_{1} p_{2}^{3}+3 q_{2}^{2}\left(4 q_{1}^{2} p_{2}^{2}+12 q_{1} q_{2} p_{1} p_{2}-3 q_{2}^{2} p_{1}^{2}\right)+16 q_{1}^{3} q_{2}^{5}, \\
& I_{2}=162 p_{1}^{3}\left(q_{1} p_{2}-q_{2} p_{1}\right)-9 q_{1}^{3}\left(4 q_{1}^{2} p_{2}^{2}-20 q_{1} q_{2} p_{1} p_{2}+13 q_{2}^{2} p_{1}^{2}\right)+16 q_{1}^{6} q_{2}^{3} .
\end{aligned}
$$

Moreover we found that for some rational and irrational values of $\alpha$ one polynomial first integral exists. This is the case for $\alpha \in\{0,-7 / 2,-4,-5,-10,-11,(-38+21 \sqrt{3}) / 11,-187 / 7\}$. For these values of $\alpha$ first integrals are quite complicated as it is shown by some examples below:

$$
\begin{array}{rlrl}
\alpha=-\frac{7}{2}, & & I=2 p_{1}\left(q_{1} p_{2}-q_{2} p_{1}\right)+q_{1}^{3} q_{2}^{2}, \\
\alpha=-4, & & I=4 p_{1}^{3}-q_{1}^{3}\left(q_{1} p_{2}-4 q_{2} p_{1}\right), \\
\alpha=-5, & I & =6 p_{1}^{3} p_{2}+q_{1}^{2}\left(q_{1}^{2} p_{2}^{2}-4 q_{1} q_{2} p_{1} p_{2}+15 q_{2}^{2} p_{1}^{2}\right)+2 q_{1}^{5} q_{2}^{3}, \\
\alpha=\frac{-38+21 \sqrt{3}}{11}, & & I=176(38+21 \sqrt{3}) p_{1}^{3} p_{2}^{3}-33\left[(39+23 \sqrt{3}) q_{1}^{4} p_{2}^{4}-4(39+23 \sqrt{3}) q_{1}^{3} q_{2} p_{1} p_{2}^{3}\right. \\
& & & \left.-10(7+3 \sqrt{3}) q_{1}^{2} q_{2}^{2} p_{1}^{2} p_{2}^{2}+4(-3+5 \sqrt{3}) q_{1} q_{2}^{3} p_{1}^{3} p_{2}+(3-5 \sqrt{3}) q_{2}^{4} p_{1}^{4}\right] \\
& & +264 q_{1}^{3} q_{2}^{3}\left[3(2+\sqrt{3}) q_{1}^{2} p_{2}^{2}-2 q_{1} q_{2} p_{1} p_{2}-3(-2+\sqrt{3}) q_{2}^{2} p_{1}^{2}\right] \\
& & -32(-7+3 \sqrt{3}) q_{1}^{6} q_{2}^{6} .
\end{array}
$$




\subsection{Case $k>4$}

We performed an analysis of the case with $k=5$ in the same way as for the case with $k=4$ in the previous section. It does not give any intersting examples of Newton's equations except for a case when the force admits only one simple Darboux point (see the last subsection) or when they do not admit any Darboux point. It should be mentioned that the bigger number of parameters make the analysis more complicated.

Below we describe a few general facts which are valid for arbitrary $k>5$. Let us consider a case when the force has exactly $k$ Darboux points. Then for each $k$ set $\mathcal{I}_{k, k}$ contains two elements

$$
\mathcal{I}_{k, k}^{(1)}=\{-1,-1, \underbrace{k-2, \ldots, k-2}_{k-2 \text { times }}\}, \quad \mathcal{I}_{k, k}^{(2)}=\{-\frac{k+1}{2 k}, \underbrace{k+1, \ldots, k+1}_{k-1 \text { times }}\} .
$$

Let us note that for $k=14,17,19,26, \ldots$ this set is bigger, see [30]. We show the following fact.

Lemma 7.2. Let $\boldsymbol{F}=\left(F_{1}, F_{2}\right)$ be a homogeneous force possesing $k$ Darboux points, where $k=1+\operatorname{deg} F_{i}$. Assume that $\left(\Lambda_{1}, \ldots, \Lambda_{k}\right)=\mathcal{I}_{k, k}^{(2)}$, where $\Lambda_{i}$ is the non-trivial Kovalevskaya exponent of $\boldsymbol{F}$ at $i$-th Darboux point. Then $\boldsymbol{F}$ is equivalent to the following force

$$
\begin{aligned}
& F_{1}=\sum_{i=1}^{k-1} i g_{i} q_{1}^{k-i} q_{2}^{i-1}, \\
& F_{2}=\sum_{i=0}^{k-1}(k+1+i) g_{i} q_{1}^{k-1-i} q_{2}^{i} .
\end{aligned}
$$

Proof. We can assume that one Darboux point is at infinity. The polynomial $g(z)$ has degree $k-1$ and the degree of polynomial $h(z)$ is smaller than $k-1$, see formulae (6.5). We put

$$
g(z)=\sum_{i=0}^{k-1} g_{i} z^{i}=g_{k-1} \prod_{i=1}^{k-1}\left(z-z_{i}\right)
$$

Then

$$
\frac{h(z)}{g(z)}=\sum_{i=1}^{k-1} \frac{g^{\prime}\left(z_{i}\right)}{h\left(z_{i}\right)} \frac{1}{z-z_{i}}=\sum_{i=1}^{k-1} \frac{1}{\Lambda_{i}} \frac{1}{z-z_{i}} .
$$

Since $\Lambda_{1}=\cdots=\Lambda_{k-1}=k+1$, we have

$$
h(z)=\frac{1}{k+1} \sum_{i=1}^{k-1}\left(\frac{1}{z-z_{i}}\right) g(z)=\frac{1}{k+1} g^{\prime}(z) .
$$

As result

$$
f_{1}(z)=\frac{1}{k+1} g^{\prime}(z), \quad f_{2}(z)=g(z)+z h(z)=g(z)+\frac{1}{k+1} z g^{\prime}(z) .
$$


Direct substitution of the explicit form of $g(z)$ yields

$$
\begin{aligned}
& f_{1}=\frac{1}{k+1} \sum_{i=1}^{k-1} i g_{i} z^{i-1}, \\
& f_{2}=\frac{1}{k+1} \sum_{i=0}^{k-1}(k+1+i) g_{i} z^{i},
\end{aligned}
$$

and homogenization of $\left(f_{1}, f_{2}\right)$ and multiplication by $k+1$ finishes the proof.

One can check directly that

$$
I=(k+1) p_{1}\left(q_{1} p_{2}-q_{2} p_{1}\right)+\sum_{i=1}^{k} g_{i-1} q_{1}^{k+2-i} q_{2}^{i-1},
$$

is a first integral of Newton's equations with force (7.29). For this force the necessary integrability conditions of Theorem 1.3 Morales-Ramis theory are satisfied. Thus we can only apply a direct method for searching the second first integral. Here the problem is not trivial as force (7.29) depends on many parameters. We have not been able to make a general search for first integrals of degree higher than 2 with respect to momenta. In all cases when a first integral was found, the system appeared to be Hamiltonian or trivially integrable.

Lemma 7.3. Let $\boldsymbol{F}=\left(F_{1}, F_{2}\right)$ be a homogeneous force possesing $k$ Darboux points, where $k=1+\operatorname{deg} F_{i}$. Assume that $\left(\Lambda_{1}, \ldots, \Lambda_{k}\right)=\mathcal{I}_{k, k}^{(1)}$, where $\Lambda_{i}$ is the non-trivial Kovalevskaya exponent of $\boldsymbol{F}$ at $i$-th Darboux point. Then $\boldsymbol{F}$ is equivalent to the following force

$$
F_{1}=\sum_{i=1}^{k-1}(i+1-k) g_{i} q_{1}^{k-i} q_{2}^{i-1}, \quad F_{2}=\sum_{i=1}^{k-1}(i-1) g_{i} q_{1}^{k-i-1} q_{2}^{i} .
$$

Proof. We can assume that one Darboux point is at the infinity and for it $\Lambda_{0}=-1$. The other with $\Lambda_{k-1}=\Lambda_{-}=-1$ can be locate at $z_{k-1}=0$. For Darboux points $z_{1}, \ldots, z_{k-2}$ we have $\Lambda_{1}=\cdots=\Lambda_{k-2}=\Lambda=k-2$. Thus

$$
\frac{h}{g}=\frac{1}{\Lambda} \sum_{i=1}^{k-2} \frac{1}{z-z_{i}}+\frac{1}{\Lambda_{-}} \frac{1}{z-z_{k-1}}=\frac{1}{\Lambda} \sum_{i=1}^{k-1} \frac{1}{z-z_{i}}+\left(\frac{1}{\Lambda_{-}}-\frac{1}{\Lambda}\right) \frac{1}{z-z_{k-1}},
$$

or, in other words

$$
h=\frac{1}{\Lambda} g^{\prime}+\left(\frac{1}{\Lambda_{-}}-\frac{1}{\Lambda}\right) \frac{g}{z-z_{k-1}}=\frac{g^{\prime}(z)}{k-2}-\frac{(k-1) g(z)}{(k-2)\left(z-z_{k-1}\right)} .
$$

We recall that $f_{1}=h$ and $f_{2}=g+z h$. Thus, putting $z_{k-1}=0$ and multiplying by $k-2$, we obtain

$$
f_{1}(z)=g^{\prime}(z)-\frac{(k-1) g(z)}{z}, \quad f_{2}(z)=z g^{\prime}(z)-g(z) .
$$

Substitution of (7.30) into the above expressions and homogenization gives (7.31). 
For force (7.31) the necessary integrability conditions of Theorem 1.3 are satisfied. Hence, as in the previous case, we can only apply a direct search for two first integrals, however conclusions from such an investigation are the same as for force (7.29).

\subsection{An integrable family of Newton equations}

Here we consider a force given by

$$
F_{1}=\lambda q_{1} q_{2}^{k-2}, \quad F_{2}=q_{2}^{k-1}, \quad \lambda \in \mathbb{C}^{\star}, \quad k>2 .
$$

This force has one Darboux point at infinity, and it satisfies the necessary conditions for integrability of Theorem 1.3 provided $\lambda$ belongs to an item of table 1.13. It appears that for all these values of $\lambda$, the Newton equations are really integrable. Moreover, this integrability is highly non-trivial.

Force (7.32) admits one obvious first integral

$$
I_{1}=\frac{1}{2} p_{2}^{2}+\frac{1}{k} q_{2}^{2}
$$

Assume that

$$
\lambda=p+\frac{k}{2} p(p-1), \quad p \in \mathbb{Z}^{\star} .
$$

Then for each $k$ and $p$ there exists an additional first integral of degree $p$ with respect to the momenta for $p>0$, for $p<0$ this degree is $1-p$. For example, if $k=3$ and $p=6$ this first integral reads

$$
I_{2}=28 p_{2}^{5}\left(q_{1} p_{2}-q_{2} p_{1}\right)-70 q_{2}^{3} p_{2}^{3}\left(6 q_{1} p_{2}-q_{2} p_{1}\right)+15 q_{2}^{6} p_{2}\left(21 q_{1} p_{2}-q_{2} p_{1}\right)-15 q_{1} q_{2}^{9},
$$

while for $k=6$ and $p=-5$ it takes the form

$$
I_{2}=11 p_{1} p_{2}^{5}+11 q_{2}^{5} p_{2}^{3}\left(17 q_{1} p_{2}-2 q_{2} p_{1}\right)-3 q_{2}^{11} p_{2}\left(34 q_{1} p_{2}-q_{2} p_{1}\right)+3 q_{1} q_{2}^{17} .
$$

We checked the above claim finding explicit forms of integrals for $k<10$ and $|p|<10$. Let us note that for natural Hamiltonian systems with a homogeneous potential in all known integrable cases the additional first integral is of the degree smaller than 5 with respect to the momenta.

If we assume that

$$
\lambda=\frac{1}{2}\left[\frac{k-1}{k}+k p(p+1)\right], \quad p \in \mathbb{N} \cup\{0\},
$$

the force admits two additional first integrals, so the corresponding Newton equations are superintegrable with three polynomial first integrals. Degrees of two additional integrals with respect to the momenta depend on $p$ and are unbounded with respect to $p$. For example, for $k=3$ and $p=0$ the additional first integrals read

$$
I_{2}=6 p_{1}\left(q_{1} p_{2}-q_{2} p_{1}\right)+q_{1}^{2} q_{2}^{2}, \quad I_{3}=6 p_{1}^{3}-q_{1}^{2}\left(q_{1} p_{2}-3 q_{2} p_{1}\right),
$$


while for $k=4$ and $p=1$ they have the following forms

$$
\begin{aligned}
I_{2} & =240 p_{1} p_{2}^{2}\left(q_{1} p_{2}-q_{2} p_{1}\right)+2 q_{2}^{3}\left(175 q_{1}^{2} p_{2}^{2}-260 q_{1} q_{2} p_{1} p_{2}+4 q_{2}^{2} p_{1}^{2}\right)-225 q_{1}^{2} q_{2}^{7}, \\
I_{3} & =10368 p_{1}^{4} p_{2}^{2}+16\left(625 q_{1}^{4} p_{2}^{4}-2500 q_{1}^{3} q_{2} p_{1} p_{2}^{3}+3750 q_{1}^{2} q_{2}^{2} p_{1}^{2} p_{2}^{2}+1280 q_{1} q_{2}^{3} p_{1}^{3} p_{2}+4 q_{2}^{4} p_{1}^{4}\right) \\
& -1350 q_{1}^{2} q_{2}^{4}\left(25 q_{1}^{2} p_{2}^{2}-80 q_{1} q_{2} p_{1} p_{2}-8 q_{2}^{2} p_{1}^{2}\right)+50625 q_{1}^{4} q_{2}^{8} .
\end{aligned}
$$

We checked the above claim as the previous one, finding explicit forms of first integrals. In all cases we checked that three first integrals $I_{1}, I_{2}$ and $I_{3}$ are functionally independent.

For $k>5$ the considered above two choices for $\lambda$ are the only admitted by Theorem 1.3 .

For $k \leq 5$ we have additional admissible choices for $\lambda$. For example, for $k=3$ the four additional families depending on integer parameter $p$ appear. Below we show first integrals for a few elements of these families: for $\lambda=-\frac{1}{24}+\frac{1}{6}(1+3 p)^{2}$

$$
\begin{array}{ll}
p=0, \quad & I_{2}=192 p_{1}^{4}-16 q_{1}^{2} p_{1}\left(q_{1} p_{2}-3 q_{2} p_{1}\right)-q_{1}^{4} q_{2}^{2}, \\
& I_{3}=192 p_{1}^{3}\left(q_{1} p_{2}-q_{2} p_{1}\right)+2 q_{1}^{2}\left(q_{1}^{2} p_{2}^{2}-4 q_{1} q_{2} p_{1} p_{2}+24 q_{2}^{2} p_{1}^{2}\right)+q_{1}^{4} q_{2}^{3}, \\
p=1, \quad & I_{2}=5184 p_{1}^{4} p_{2}^{2}-16 p_{1}\left(343 q_{1}^{3} p_{2}^{3}-1029 q_{1}^{2} q_{2} p_{1} p_{2}^{2}-672 q_{1} q_{2}^{2} p_{1}^{2} p_{2}+8 q_{2}^{3} p_{1}^{3}\right) \\
& -147 q_{1}^{2} q_{2}^{2}\left(49 q_{1}^{2} p_{2}^{2}-224 q_{1} q_{2} p_{1} p_{2}-32 q_{2}^{2} p_{1}^{2}\right)+14406 q_{1}^{4} q_{2}^{5}, \\
& I_{3}=36288 p_{1}^{3} p_{2}^{4}\left(q_{1} p_{2}-q_{2} p_{1}\right)+14 p_{2}^{2}\left(343 q_{1}^{4} p_{2}^{4}-1372 q_{1}^{3} q_{2} p_{1} p_{2}^{3}+12264 q_{1}^{2} q_{2}^{2} p_{1}^{2} p_{2}^{2}\right. \\
& \left.-12928 q_{1} q_{2}^{3} p_{1}^{3} p_{2}+640 q_{2}^{4} p_{1}^{4}\right)-q_{2}^{3}\left(7203 q_{1}^{4} p_{2}^{4}-208544 q_{1}^{3} q_{2} p_{1} p_{2}^{3}+272832 q_{1}^{2} q_{2}^{2} p_{1}^{2} p_{2}^{2}\right. \\
& \left.-16128 q_{1} q_{2}^{3} p_{1}^{3} p_{2}-256 q_{2}^{4} p_{1}^{4}\right)+588 q_{1}^{2} q_{2}^{6}\left(147 q_{1}^{2} p_{2}^{2}-280 q_{1} q_{2} p_{1} p_{2}+16 q_{2}^{2} p_{1}^{2}\right)-28812 q_{1}^{4} q_{2}^{9},
\end{array}
$$

for $\lambda=-\frac{1}{24}+\frac{3}{32}(1+4 p)^{2}$

$$
\begin{aligned}
p=0, \quad & I_{2}=294912 p_{1}^{6}+15360 q_{1}^{2} p_{1}^{3}\left(-q_{1} p_{2}+3 q_{2} p_{1}\right)-16 q_{1}^{4}\left(q_{1}^{2} p_{2}^{2}-6 q_{1} q_{2} p_{1} p_{2}+90 q_{2}^{2} p_{1}^{2}\right)-9 q_{1}^{6} q_{2}^{3}, \\
& I_{3}=28311552 p_{1}^{7}\left(q_{1} p_{2}-q_{2} p_{1}\right)+516096 q_{1}^{2} p_{1}^{4}\left(q_{1}^{2} p_{2}^{2}-4 q_{1} q_{2} p_{1} p_{2}+16 q_{2}^{2} p_{1}^{2}\right. \\
& +1536 q_{1}^{4} p_{1}\left(-q_{1}^{3} p_{2}^{3}+7 q_{1}^{2} q_{2} p_{1} p_{2}^{2}+14 q_{1} q_{2}^{2} p_{1}^{2} p_{2}+84 q_{2}^{3} p_{1}^{3}\right)-8 q_{1}^{6} q_{2}^{2}\left(5 q_{1}^{2} p_{2}^{2}+132 q_{1} q_{2} p_{1} p_{2}\right. \\
& \left.-1008 q_{2}^{2} p_{1}^{2}\right)-27 q_{1}^{8} q_{2}^{5} .
\end{aligned}
$$

For $\lambda=-\frac{1}{24}+\frac{3}{50}(1+5 p)^{2}$ and for $\lambda=-\frac{1}{24}+\frac{3}{50}(2+5 p)^{2}$ expressions for $I_{2}$ and $I_{3}$ are more complicated and we do not write them here.

Our calculations show that for additional families of $\lambda$ 's there always exist two additional functionally independent first integrals.

The investigations shortly presented above allowed us to formulate Conjecture 1.1. Without doubt, the integrability analysis of equations (1.17) presented above is not satisfactory, and a separate paper [23] will be devoted to more involved investigations.

\section{Appendix A}

Here we characterize in two ways forces that are potential in the sense of Definition 2 ,

Lemma A.4. Assume that Newton's equations

$$
\dot{\boldsymbol{q}}=\boldsymbol{p}, \quad \dot{\boldsymbol{p}}=-\boldsymbol{F}(\boldsymbol{q}), \quad \boldsymbol{q}, \boldsymbol{p} \in \mathbb{C}^{n},
$$


admit a first integral of the form

$$
I=\frac{1}{2} \boldsymbol{p}^{T} K \boldsymbol{p}+W(\boldsymbol{q}),
$$

where $K \in \operatorname{GL}(n, \mathbb{C}), K=K^{T}$. Then force $\boldsymbol{F}$ is potential and equations (A.1) are Hamiltonian with respect to a symplectic structure given by the following matrix

$$
J=\left[\begin{array}{cc}
\mathbf{0} & K^{-1} \\
-K^{-1} & \mathbf{0}
\end{array}\right]
$$

and I becomes the Hamiltonian function.

Proof. Since $I$ is a first integral of equations (A.1), we have

$$
\boldsymbol{p}^{T} \frac{\partial W}{\partial \boldsymbol{q}}+\boldsymbol{p}^{T} K \boldsymbol{F}=0
$$

so

$$
\boldsymbol{F}=K^{-1} \frac{\partial W}{\partial \boldsymbol{q}}=K^{-1} \frac{\partial I}{\partial \boldsymbol{q}}
$$

Hence

$$
\left[\begin{array}{c}
\boldsymbol{p} \\
-\boldsymbol{F}
\end{array}\right]=\left[\begin{array}{cc}
\mathbf{0} & K^{-1} \\
-K^{-1} & \mathbf{0}
\end{array}\right]\left[\begin{array}{l}
\frac{\partial I}{\partial \boldsymbol{q}} \\
\frac{\partial I}{\partial \boldsymbol{p}}
\end{array}\right]
$$

In this way we proved the second claim of our lemma. To prove the first one, let us notice that for every non-singular matrix $K$ there exists a matrix $A \in \operatorname{GL}(n, \mathbb{C})$ such that $K=A A^{T}$. Hence, in new variables

$$
\boldsymbol{Q}=A \boldsymbol{q}, \quad \boldsymbol{P}=A \boldsymbol{p}, \quad \text { where } \quad A A^{T}=K,
$$

equations (A.1) read

$$
\dot{\boldsymbol{Q}}=\boldsymbol{P}, \quad \dot{\boldsymbol{P}}=-\nabla V(\boldsymbol{Q}), \quad V(\boldsymbol{Q}):=W\left(A^{-1} \boldsymbol{Q}\right),
$$

so they are canonical Hamilton's equations with

$$
H:=I\left(A^{-1} \boldsymbol{Q}, A^{-1} \boldsymbol{P}\right)=\frac{1}{2} \boldsymbol{P}^{T} \boldsymbol{P}+V(\boldsymbol{Q}),
$$

as the Hamiltonian function.

The second characterization of potential forces is the following. If a force $\boldsymbol{F}(\boldsymbol{q})$ is potential, then, by Definition 2, there exists a non-singular matrix $A$ such that

$$
\boldsymbol{F}_{A}(\boldsymbol{Q})=A^{-1} \boldsymbol{F}(A \boldsymbol{Q})=\frac{\partial V(Q)}{\partial Q},
$$


for some scalar function $V$. Hence, putting $\boldsymbol{q}=A \boldsymbol{Q}$ we obtain

$$
K \boldsymbol{F}(\boldsymbol{q})=\frac{\partial V(\boldsymbol{q})}{\partial \boldsymbol{q}}, \quad K^{-1}=A^{T} A
$$

This shows that $\boldsymbol{F}$ is a potential iff there exists a non-singular matrix $K$ such that differential form

$$
w=\langle K \boldsymbol{F}, \mathrm{d} \boldsymbol{q}\rangle:=\sum_{i=1}^{n} \sum_{j=1}^{n} K_{i j} F_{j} \mathrm{~d} q_{i},
$$

is exact.

The condition $\mathrm{d} w=0$ is equivalent to the following set of equations

$$
\sum_{i=1}^{n}\left(K_{p i} \frac{\partial F_{i}}{\partial q_{r}}-K_{r i} \frac{\partial F_{i}}{\partial q_{p}}\right)=0, \quad 1 \leq p<r \leq n .
$$

For a given force in the above equations the elements of matrix are the unknowns $K=\left[K_{i j}\right]$ with $i \leq j$. Since we consider the polynomial force and $\operatorname{deg} F_{i}=k-1$, all $n(n-1) / 2$ equations (A.9) are polynomial and homogeneous of degree $k-2$, Hence, they give rise to a system of homogeneous linear equations for unknowns $K_{i j}$.

As an example we consider the case $n=2$ and $k=3$. We put

$$
F_{1}=\frac{a_{11}}{2} q_{1}^{2}+a_{12} q_{1} q_{2}+\frac{a_{13}}{2} q_{2}^{2}, \quad F_{2}=\frac{a_{21}}{2} q_{1}^{2}+a_{22} q_{1} q_{2}+\frac{a_{23}}{2} q_{2}^{2} .
$$

Then equations (A.9) are equivalent to the following linear system

$$
L \boldsymbol{k}=0, \quad L=\left(\begin{array}{lll}
a_{13} & a_{23}-a_{12} & -a_{22} \\
a_{12} & a_{22}-a_{11} & -a_{21}
\end{array}\right)
$$

and $\boldsymbol{k}=\left[K_{11}, K_{12}, K_{22}\right]^{T}$. This system has always a non-zero solution. If one of $2 \times 2$ minors $L_{i}$ of matrix $L$

$L_{1}=a_{12}\left(a_{12}-a_{23}\right)+a_{13}\left(a_{22}-a_{11}\right), L_{2}=a_{21}\left(a_{12}-a_{23}\right)+a_{22}\left(a_{22}-a_{11}\right), L_{3}=a_{12} a_{22}-a_{13} a_{21}$, is non-zero, then it has the follwing solution

$$
\begin{aligned}
& K_{11}=a_{21}\left(a_{12}-a_{23}\right)+a_{22}\left(a_{22}-a_{11}\right), \quad K_{12}=a_{13} a_{21}-a_{12} a_{22}, \\
& K_{22}=a_{13}\left(a_{22}-a_{11}\right)+a_{12}\left(a_{12}-a_{23}\right),
\end{aligned}
$$

and matrix $K$ given by this solution is not singular iff

$$
\begin{aligned}
& a_{12}^{2}\left[a_{12} a_{21}-a_{11} a_{22}-2 a_{21} a_{23}\right]+a_{12}\left[\left(a_{11}-a_{22}\right) a_{22} a_{23}-a_{13} a_{21}\left(a_{11}-3 a_{22}\right)+a_{21} a_{23}^{2}\right] \\
& +a_{13}\left[\left(a_{11}-a_{22}\right)\left(\left(a_{11}-a_{22}\right) a_{22}+a_{21} a_{23}\right)-a_{13} a_{21}^{2}\right] \neq 0 .
\end{aligned}
$$

For values of $a_{i j}$ for which all minors $L_{i}$ vanish one can find a two-parameter family of solutions of (A.11) and always obtain a non-singular matrix $K$.

For $n=2$ and $k>3$, as well as, for $n>2$ equations (A.9) give rise to an overdetermined system of linear homogeneous equations, so a non-zero solution exists only if the rank of matrix $L$ is not maximal. As entries of $L$ depend on coefficients of polynomials $F_{1}$ and $F_{2}$, this gives very restrictive conditions for a force $\boldsymbol{F}=\left(F_{1}, F_{2}\right)$ to be potential. 


\section{Appendix B}

Assume that we know a particular solution $\varphi(t)$ of a holomorphic system

$$
\frac{\mathrm{d}}{\mathrm{d} t} \boldsymbol{x}=\boldsymbol{v}(\boldsymbol{x}), \quad \boldsymbol{x} \in \mathbb{C}, \quad t \in \mathbb{C} .
$$

Then appart from variational equations

$$
\frac{\mathrm{d}}{\mathrm{d} t} \boldsymbol{\xi}=A(t) \boldsymbol{\xi}, \quad A(t)=\frac{\partial \boldsymbol{v}}{\partial \boldsymbol{x}}(\boldsymbol{\varphi}(t)),
$$

one can use higher order variational equations. The idea is following. Let us put

$$
\boldsymbol{x}=\boldsymbol{\varphi}(t)+\varepsilon \boldsymbol{\xi}^{(1)}+\varepsilon^{2} \boldsymbol{\xi}^{(2)}+\cdots+\varepsilon^{k} \boldsymbol{\xi}^{(k)}+\cdots,
$$

where $\varepsilon$ is a formal small parameter. Inserting the above expansion into equation (B.1) and comparing terms of the same order with respect to $\varepsilon$, we obtain the following chain of linear non-homogeneous equations

$$
\frac{\mathrm{d}}{\mathrm{d} t} \boldsymbol{\xi}^{(k)}=A(t) \boldsymbol{\xi}^{(k)}+\boldsymbol{f}_{k}\left(\boldsymbol{\xi}^{(1)}, \ldots, \boldsymbol{\xi}^{(k-1)}\right), \quad k=1,2, \ldots,
$$

where $\boldsymbol{f}_{1} \equiv 0$. For a given $k$ equation $(\overline{B .3})$ is called the $k$-th order variational equation. We denote by $X(t)$ the fundamental matrix of the homogeneous system, i.e., $n \times n$ matrix satisfying

$$
\frac{\mathrm{d}}{\mathrm{d} t} X=A(t) X, \quad X(0)=E,
$$

where $E$ is the identity matrix. Then solutions of $k$-th order variational equations for $k>1$ are given by

$$
\boldsymbol{\xi}^{(k)}(t)=X(t) \boldsymbol{c}(t),
$$

where $\boldsymbol{c}(t)$ is a solution of

$$
\frac{\mathrm{d}}{\mathrm{d} t} \boldsymbol{c}=X^{-1}(t) \boldsymbol{f}_{k} .
$$

There exists an appropriate framework allowing to define the differential Galois group of the $k$-th order variational equation, for details see [24; 27]. For Hamiltonian systems the following theorem was proved in [27].

Theorem B.1. Assume that a Hamiltonian system is meromorphically integrable in the Liouville sense in a neighborhood of a phase curve $\Gamma$ which is not an equilibrium point. Then the identity component of the differential Galois group of the $k$-th order variational equations is Abelian for any $k \in \mathbb{N}$.

The main problem with an application of the above theorem lies in the fact that it is very difficult to investigate the differential Galois group of higher order variational equations. However, if the first order variational equations are a direct product of Lamé equations, then there is a local criterion which allows to decide whethere this group is Abelian, for details and practical applications see $24 ; 27 ; 20 ; 22]$. 
Let us note that the main construction presented in [27] is valid also for non-Hamiltonian systems. Thus, we can also use it for the Newton's equations. Unfortunately, there are many theoretical problems in extending Theorem B.1 in such way that it should apply to the Newton equations. Nevertheless, there are many facts strongly suggesting that the following conjecture is true.

Conjecture B.1. Assume that a Newton system of the form (1.5) is meromorphically integrable in the Jacobi sense in a neighborhood of a phase curve $\Gamma$ corresponding to a Darboux point. Then the identity component of the differential Galois group of the $k$-th order variational equations is Abelian for any $k \in \mathbb{N}$.

\section{Acknowledgments}

The author is very grateful to Andrzej Maciejewski for many helpful comments and suggestions concerning improvements and simplifications of some results. The author also thanks Alain Albouy for his fruitful comments about quasi-Lagrangian systems and sending Halphen's papers. This research has been partially supported by the European Community project GIFT (NEST-Adventure Project no. 5006) and by projet de l'Agence National de la Recherche "Intégrabilité réelle et complexe en mécanique hamiltonienne" $\mathrm{N}^{\circ} \mathrm{JC} 05_{-} 41465$. .

\section{References}

[1] Arnold, V. I., Mathematical Methods of Classical Mechanics, Graduate Texts in Mathematics, Springer-Verlag, New York, 1978.

[2] Arnold, V. I., Kozlov, V. V., and Neishtadt, A. I., Dynamical systems. III, volume 3 of Encyclopaedia of Mathematical Sciences, Springer-Verlag, Berlin-New York, 1988.

[3] Audin, M., Les systèmes hamiltoniens et leur intégrabilité, Cours Spécialisés 8, Collection SMF, SMF et EDP Sciences, Paris, 2001.

[4] Baider, A., Churchill, R. C., Rod, D. L., and Singer, M. F., On the infinitesimal geometry of integrable systems, in Mechanics day (Waterloo, ON, 1992), volume 7 of Fields Inst. Commun., pages 5-56, Amer. Math. Soc., Providence, RI, 1996.

[5] Chaplygin, S. A., Izbrannye trudy [Selected works], Izdat. "Nauka", Moscow, 1976.

[6] Churchill, R. C., Two generator subgroups of SL $(2, \mathbf{C})$ and the hypergeometric, Riemann, and Lamé equations, J. Symbolic Comput., 28(4-5):521-545, 1999, differential algebra and differential equations.

[7] Fedorov, Y. N. and Kozlov, V. V., A Memoir on Integrable Systems, Springer Verlag, 2006, to appear. 
[8] Gallavotti, G., The elements of mechanics, Texts and Monographs in Physics, Springer-Verlag, New York, 1983.

[9] Griffiths, P. and Harris, J., Principles of algebraic geometry, Wiley-Interscience [John Wiley \& Sons], New York, 1978.

[10] Guillot, A., Champs quadratiques uniformisables, Ph.D. thesis, Ècole Normale Supérieure de Lyon, France, 2001.

[11] Guillot, A., Un théorème de point fixe pour les endomorphismes de l'espace projectif avec des applications aux feuilletages algébriques, Bull. Braz. Math. Soc. (N.S.), $35(3): 345-362,2004$.

[12] Iwasaki, K., Kimura, H., Shimomura, S., and Yoshida, M., From Gauss to Painlevé, A modern theory of special functions, Aspects of Mathematics, E16, Friedr. Vieweg \& Sohn, Braunschweig, 1991.

[13] Jacobi, C. G. J., Vorlesungen über Dynamik, Bruck und Velag von G. Reimer, Berlin, 1884.

[14] Jovanović, B., Geometry and integrability of Euler-Poincaré-Suslov equations, Nonlinearity, 14(6):1555-1567, 2001.

[15] Kaplansky, I., An introduction to differential algebra, Actualités Scientifiques et Industrielles, No. 1251, Publications de l'Institut de Mathématique de l'Université de Nancago, No. V, Hermann, Paris, second edition, 1976.

[16] Kimura, T., On Riemann's equations which are solvable by quadratures, Funkcial. Ekvac., 12:269-281, 1969/1970.

[17] Kozlov, V. V., Symmetries, Topology and Resonances in Hamiltonian Mechanics, Springer-Verlag, Berlin, 1996.

[18] Maciejewski, A. J., Ollangnier, J. M., Nowicki, A., and Strelcyn, J.-M., Around Jouanolou non-integrability theorem, Indag. Math., N.S., 11(2):239-254, 2000.

[19] Maciejewski, A. J. and Przybylska, M., Non-integrability of ABC flow, Phys. Lett. A, 303:265-272, 2002.

[20] Maciejewski, A. J. and Przybylska, M., All meromorphically integrable 2D Hamiltonian systems with homogeneous potential of degree 3, Phys. Lett. A, 327(5-6):461-473, 2004.

[21] Maciejewski, A. J. and Przybylska, M., Nonintegrability of the Suslov problem., J. Math. Phys., 45(3):1065-1078, 2004.

[22] Maciejewski, A. J. and Przybylska, M., Darboux points and integrability of Hamiltonian systems with homogeneous polynomial potential, J. Math. Phys., 46(6):062901, 33 pp., 2005. 
[23] Maciejewski, A. J. and Przybylska, M., About some class of integrable Newton equations without bound on degree of first integrals, 2007, in preparation.

[24] Morales Ruiz, J. J., Differential Galois theory and non-integrability of Hamiltonian systems, volume 179 of Progress in Mathematics, Birkhäuser Verlag, Basel, 1999.

[25] Morales-Ruiz, J. J. and Ramis, J. P., Galoisian obstructions to integrability of Hamiltonian systems: statements and examples, in Hamiltonian systems with three or more degrees of freedom (S'Agaró, 1995), volume 533 of NATO Adv. Sci. Inst. Ser. C Math. Phys. Sci., pages 509-513, Kluwer Acad. Publ., Dordrecht, 1999.

[26] Morales-Ruiz, J. J. and Ramis, J. P., Galoisian obstructions to integrability of Hamiltonian systems. I, Methods Appl. Anal., 8(1):33-95, 2001.

[27] Morales-Ruiz, J. J., Ramis, J. P., and Simó, C., Integrability of Hamiltonian systems and differential Galois groups of higher variational equations, 2006, to appear in Ann. Sci. École Norm. Sup., available at http://www.maia.ub.es/dsg/2005/0506simo.pdf.

[28] Newton, I., Philosophiae Naturalis Principia Mathematica, S. Pepys Reg. Soc. Praeses, Londini, 1687.

[29] Poincaré, H., Les méthodes nouvelles de la mécanique céleste I, Gautier-Villars, Paris, 1892.

[30] Przybylska, M., Darboux points and integrability of Hamiltonian systems with homogeneous polynomial potential II, in preparation.

[31] Przybylska, M., Finiteness of integrable $n$-dimensional homogeneous polynomial potentials, Phys Lett A., accepted.

[32] Singer, M. F. and Ulmer, F., Galois groups of second and third order linear differential equations, J. Symbolic Comput., 16(1):9-36, 1993.

[33] van der Put, M. and Singer, M. F., Galois theory of linear differential equations, volume 328 of Grundlehren der Mathematischen Wissenschaften [Fundamental Principles of Mathematical Sciences], Springer-Verlag, Berlin, 2003.

[34] Yoshida, H., A criterion for the nonexistence of an additional integral in Hamiltonian systems with a homogeneous potential, Phys. D, 29(1-2):128-142, 1987.

[35] Ziglin, S. L., Branching of solutions and non-existence of first integrals in Hamiltonian mechanics. I, Functional Anal. Appl., 16:181-189, 1982.

[36] Ziglin, S. L., Branching of solutions and non-existence of first integrals in Hamiltonian mechanics. II, Functional Anal. Appl., 17:6-17, 1983.

[37] Ziglin, S. L., On the nonintegrability of the $A B C$-flow for $A=B$, Funktsional. Anal. i Prilozhen., 30(2):80-81, 1996. 
[38] Ziglin, S. L., The absence of a real-analytic first integral for ABC flow when $A=B$, Chaos, 8(1):272-273, 1998.

[39] Ziglin, S. L., An analytic proof of the nonintegrability of the ABC-flow for $A=B=C$, Funktsional. Anal. i Prilozhen., 37(3):77-80, 2003.

[40] Ziglin, S. L. and Cherepenin, V. A., On the nonintegrability of the ABC-flow for $A=B=C$, Dokl. Akad. Nauk, 369(2):173-174, 1999. 